Analysis of differences in the ENDL99 and ENDL2009 nuclear data libraries pertaining to $238 \mathrm{U}$

W. E. Ormand, B. Beck, I. J. Thompson, F. S. Dietrich, M. A. Descalle

October 17, 2012 
This document was prepared as an account of work sponsored by an agency of the United States government. Neither the United States government nor Lawrence Livermore National Security, LLC, nor any of their employees makes any warranty, expressed or implied, or assumes any legal liability or responsibility for the accuracy, completeness, or usefulness of any information, apparatus, product, or process disclosed, or represents that its use would not infringe privately owned rights. Reference herein to any specific commercial product, process, or service by trade name, trademark, manufacturer, or otherwise does not necessarily constitute or imply its endorsement, recommendation, or favoring by the United States government or Lawrence Livermore National Security, LLC. The views and opinions of authors expressed herein do not necessarily state or reflect those of the United States government or Lawrence Livermore National Security, LLC, and shall not be used for advertising or product endorsement purposes.

This work performed under the auspices of the U.S. Department of Energy by Lawrence Livermore National Laboratory under Contract DE-AC52-07NA27344. 


\title{
Analysis of differences in the ENDL99 and ENDL2009 nuclear data libraries pertaining to ${ }^{238} \mathbf{U}$
}

W. Erich Ormand, Bret Beck, Ian J. Thompson, Frank S. Dietrich, Marie-Anne Descalle

Lawrence Livermore National Laboratory, L-414, P.O.Box 808, Livermore, CA 94551

\begin{abstract}
A careful analysis is performed on the differences exhibited in the evaluated elastic and inelastic channels between the ENDL99 and ENDL2009 libraries for neutrons incident on ${ }^{238} \mathrm{U}$. The two libraries are compared with experimental data as well as results obtained from calculations utilizing a new Hauser-Feshbach reaction code and two modern, high-quality optical potentials. Overall, the ENDL2009 evaluation is found to be more physical, and a better representation of experimental data. The only exception is that the angular distributions for the elastic channel in the ENDL99 library are in better agreement at backward angles for incident neutron energies in the range 0.7-1.3 MeV. Other issues with the inelastic scattering data in ENDL2009 are pointed out.
\end{abstract}




\section{Contents}

I. Introduction

II. Data

III. Modeling

$\begin{array}{ll}\text { IV. Optical Potential } & 7\end{array}$

V. Total Cross Section 9

VI. Elastic Angular Distributions 11

VII. Inelastic Scattering 16

VII.1. Inelastic scattering to specified states below $E_{\text {cut }} \quad 19$

VII.2. Inelastic scattering to specified states above $E_{\text {cut }}$

VII.3. Inelastic scattering to unspecified states 24

$\begin{array}{ll}\text { VIII. Critical Assemblies } & 27\end{array}$

$\begin{array}{ll}\text { IX. Conclusions } & 29\end{array}$

X. Acknowledgements 31

A. Comments on Experimental Data Base 32

A.1. Total cross sections 33

A.2. Low-energy parameters 33

A.3. Angular distributions 34

A.4. Absorption 34

A.5. Deformation parameters 35

B. Details for optical potential

$\begin{array}{ll}\text { References } & 38\end{array}$ 


\section{INTRODUCTION}

Important differences have been identified in the nuclear data libraries ENDL99 and its recent update ENDL2009. ENDL99 is a nuclear data library with a primary emphasis on neutron-induced reactions for many target nuclei, including actinide nuclides. The evaluation was performed in the early 1990's, with an update to the ${ }^{239} \mathrm{Pu}(\mathrm{n}, 2 \mathrm{n})$ cross section in 1999. ENDL2009 is a newer evaluation primarily based on more recent experimental data and model calculations. In particular, the actinides in ENDL2009 were updated with the newer ENDF/B-VII.0 [1] and ENDF/B-VII.1 [2] evaluations. In the course of using the newer ENDL2009 evaluation, key differences between the ENDL99 and ENDL2009 libraries became apparent, with specific questions raised in the ${ }^{238} \mathrm{U}$ elastic and inelastic scattering channels. These questions deal with both the magnitude of the cross sections, as well as the angular distributions. Because of the apparent differences, and the modernity of the ENDL2009 evaluation, it is natural to ask what the origin of the differences is, and which data library better reflects the physical world. In 2012, our team was tasked with addressing both these questions. In short, the main question is which data library could be trusted and what could be done to provide greater overall confidence in the evaluated libraries. Towards this end, we searched the literature for appropriate data to test the evaluations. In addition, we developed a significant modeling capability to enhance our testing capability. This included the completion of new Hauser-Feshbach code and the development of a new optical potential for use with actinide nuclei.

The structure of this report is as follows. In Section II, we give a short description of the experimental data that we used to examine the data, with a substantially more detailed description given in Appendix A. The modeling effort is described in Section III and the optical potential is described in Section IV, and in more detail in Appendix B. The data in the ENDL99 and ENDL2009 libraries are compared with experimental data in Section V for the total cross section; Section VI for the elastic scattering data; and Section VII for the inelastic data. In Section VIII, the data libraries are used to simulate critical assemblies made with a ${ }^{238} \mathrm{U}$ reflector and compared with experimental vales of $k_{\text {eff }}$. Conclusions are gathered into Section IX. 


\section{DATA}

A critical comparison between the ENDL99 and ENDL2009 begins with selecting highquality data for the elastic and inelastic scattering channels for neutron energies ranging from $100 \mathrm{keV}$ to approximately $3 \mathrm{MeV}$. This data must include both the absolute cross section, as well as the angular distributions. Although ${ }^{238} \mathrm{U}$ has been a standard target used to study neutron reactions on actinide nuclides, there is not a plethora of high-quality data suitable for our purpose. Generally speaking, neutron experiments are difficult, leading to larger statistical uncertainties as well as poorly understood systematic uncertainties. In addition, it is often difficult to isolate the elastic and inelastic channels due to the low excitation energy of excited states in ${ }^{238} \mathrm{U}$. At higher energies, other channels open up, such as fission, that lead to larger background signals. In the course of this investigation, we have focused on a few sets of experimental data that we believe to be of sufficiently high quality to allow a critical assessment. These data provide information on the total cross section, the elastic cross section and the elastic angular distributions, and the cross section and angular distributions for inelastic scattering to the $J^{\pi}=2^{+}$and $J^{\pi}=4^{+}$excited states in ${ }^{238} \mathrm{U}$. These are listed in Table I.

TABLE I: List of data utilized in this report.

\begin{tabular}{ll}
\hline Byoun-1973 [3] & Total \\
Harvey-1988 $[4]$ & Total \\
Grigoriev-1990 [5] & Total \\
Schwartz-1974 $[6]$ & Total \\
Poenitz-1981 [7] & Total \\
Poenitz-1983 [8] & Total \\
Abfalterer-2001 [9] & Total \\
Smith-1963 [10] & Elastic (Angular dist., Legendre decomposition) \\
Haouat-1982 [11] & Elastic and Inelastic (Angular distributions) \\
\hline
\end{tabular}

A detailed explanation of data needed for an evaluation, including modeling efforts, is given in Appendix A. 


\section{MODELING}

As part of the overall check on the data, we performed several calculations using results from modern optical-model potentials fed into a Hauser-Feshbach code. The HauserFeshbach code is a new code written by W. E. Ormand. The basic idea behind the reaction modeling is that the optical potential, described in more detail in Section IV, provides both the transmission coefficients, $T_{c}$, for the particles in a channel $c$ and the cross section and angular distributions for both the "shape" elastic and direct inelastic components. The transmission coefficients determine both the absorption and decay probability. The absorption cross section is given by

$$
\sigma_{c m p d}=\pi \lambda^{2} \sum_{c} g_{c} T_{c}
$$

where $\lambda$ is the reduced wavelength for the incident particle, and the sum is over all the incident channels, $c$, e.g., particle types, angular momentum, etc. $g_{c}$ is the statistical weight in channel $c$. After the incident particle is absorbed, the system decays via the emission of particles (and photons). A key consideration is the time scale involved. If the time scale for particle emission is fairly long, then the excited nucleus has a chance to dissipate the energy given to it from the incident projectile and form the so-called compound nucleus. In this case, the nucleus may be thought of as being thermalized with excitations that are statistical in nature and decay is independent of the initial conditions. These conditions typically dominate for low incident energies (for neutrons typically $E_{n}<7 \mathrm{MeV}$ ). In this regime, the probability to decay to a state $c^{\prime}$ is given by

$$
P\left(c \rightarrow c^{\prime}\right)=\frac{T_{c^{\prime}}}{\sum_{c^{\prime \prime}} T_{c}^{\prime \prime}} .
$$

In practical calculations, the density of states becomes quite high, and above some energy, $E_{c u t}$, one dispenses with the discrete states, and, instead, uses the density of states (usually modeled), which is then accounted for with bins with width $\Delta E$. Eq. (2) is then generalized to an integral over the level density and to represent decay to the set of states within a bin. Once the time scale for particle emission shortens (this usually starts for $E_{n}>9 \mathrm{MeV}$ ), the probability that the system can decay before thermalization increases, and some fraction do. Decays of this type are usually referred to as pre-equilibrium emission. This is a different process than the Hauser-Feshbach decay and separate pre-equilibrium models need to be coupled to the Hauser-Feshbach decay. In general, the pre-equilibrium model acts before 

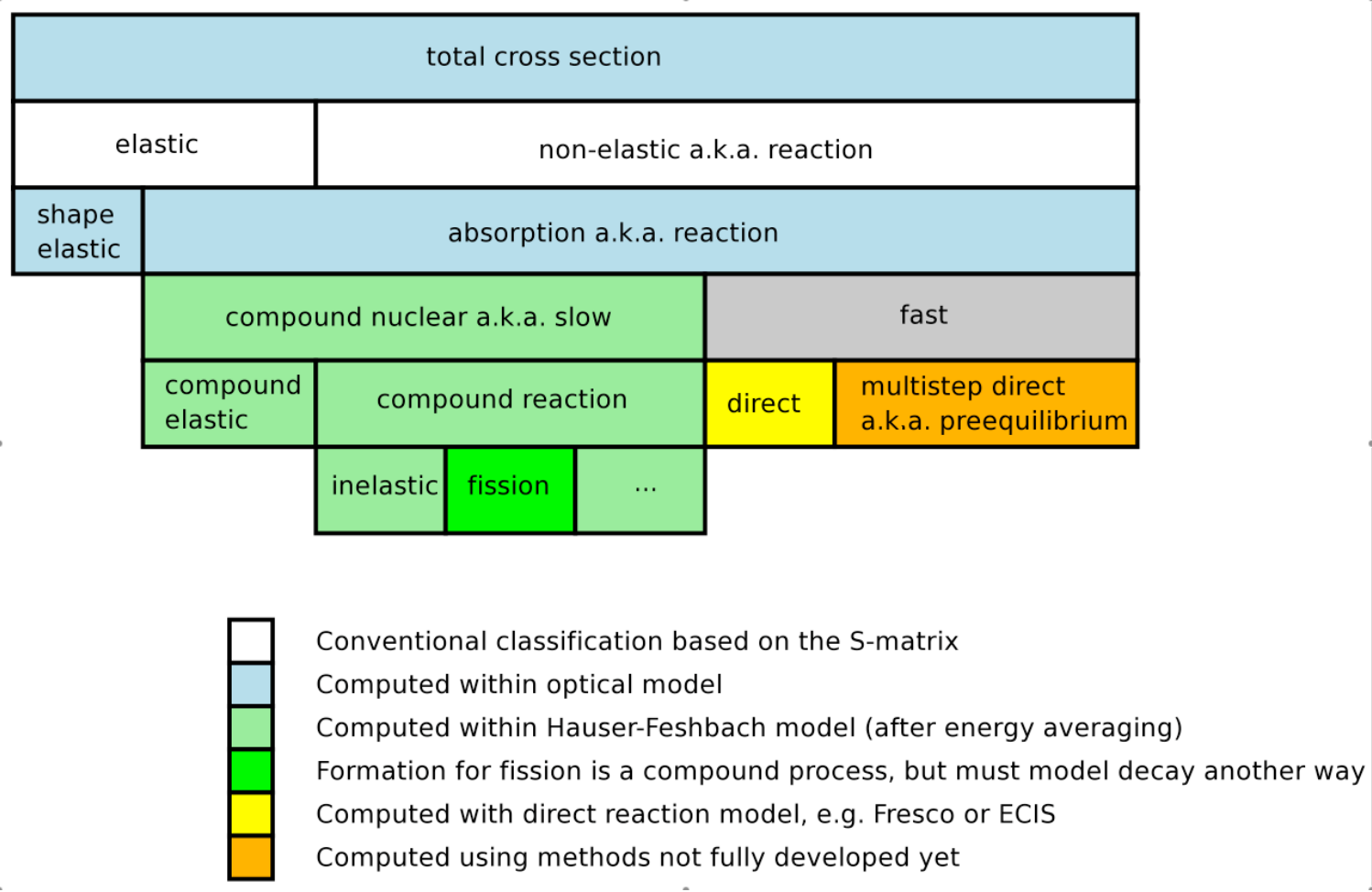

FIG. 1: Schematic of the decay processes. Each line gives a breakdown of where each channel in the reaction enters as a fraction of the total cross section.

the Hauser-Feshbach processes, and removes a portion of the total absorbed cross section from the subsequent statistical decay. A schematic of the decay process showing where various kinds of channels enter is given in Fig. 1).

The code developed here is quite general and differs somewhat from several other HauserFeshbach codes in that it performs the decays using a Monte Carlo method. This new code treats the reaction as an ensemble of initial populations of the system, and calculates the decays of each ensemble all the way to the final nucleus using Monte Carlo methods to sample all the possible exit probabilities as defined in Eq. (2). From each ensemble, the particles emitted in the decay chain are stored and analyzed event-by-event, much as in experiments. Calculations typically involved $10^{6}$ to $10^{7}$ initial ensembles in order to ensure acceptable statistical fluctuations in channels with small cross sections.

The mechanics of the decay process in the code is as follows. First, prior to the decay processes, the decay probability for every channel is pre-computed. Rather than keeping track of every single possible decay channel, only those whose individual decay probability exceeds a preset threshold, typically $10^{-6}$, is kept. It is then stored into a list contained in 
a single array as a cumulative probability from zero to unity. During the run, The decay is chosen by first selecting a random number $R$ in the range $(0,1)$ and finding the element in the array containing $R$ using a bisection search algorithm. The code was written in FORTRAN 95 and uses derived types along with dynamic memory allocation in order to optimize memory usage.

The Monte Carlo method allows for an explicit tracking of particles emitted from all channels as well as a better treatment of the angular distributions for each channel without resorting to approximations on the apportionment of the reaction cross section into the various reaction paths leading to the same final state. This is less of a problem at the very lowest energies, where only the elastic and inelastic channels are open. In this limit, it is relatively straight forward to determine both the spectra and angular distributions for the emitted particles using deterministic methods that calculate decays for the entire system by sweeping through all the states in the decay chain and accumulating their properties along the way. At higher energies, this is not feasible in a direct fashion because as the compound nucleus decays through several nuclei, it is not possible to keep track of the amount of cross section that flows into each channel separately.

This new Hauser-Feshbach code is used with the coupled-channels optical-model program ECIS06 using an interface written by F. Dietrich. In a deformed (nonspherical) nucleus such as ${ }^{238} \mathrm{U}$, the use of the coupled-channels technique is important as it allows for separation of the direct cross section (elastic and directly-coupled inelastic) arising from the spatiallydeformed optical potential from the part of the cross section coming from the decay of the compound nucleus. This impacts the lower energies studied here, where, for example, elastic neutrons emitted from the compound nucleus (decay of the compound straight to the ground state of the target) is considerable and must be added to the shape elastic process arising from the optical potential. In addition, direct inelastic scattering to states in the groundstate rotational band in ${ }^{238} \mathrm{U}$ must be combined with the component from the compound nucleus formation to these same states for a proper comparison with experiment.

\section{OPTICAL POTENTIAL}

A key ingredient to carrying out the Hauser-Feshbach modeling is the optical potential. The neutron optical potential determines the transmission coefficients for all entrance and 
exit partial waves, and hence also the compound-nucleus $(\mathrm{CN})$ production cross section $\sigma_{c m p d}$. This cross section, also known as the absorption or fusion cross section, is very difficult to measure accurately, but it is of critical importance as it is the sum of all processes (such as fission as well as particle emission) resulting from decay of the statistically equilibrated compound nucleus.

An important consideration has come to light in the past year. In order to properly account for the direct excitation of the ground-state band (i.e. not through the compound nucleus), traditionally one performs a coupled-channels calculation for a deformed system. In the past, the coupled-channels calculations were performed by including three states within the ground-state band. It has recently been shown [12] that this is insufficient as the the influence of other states in the band are not yet inconsequential. Instead, it has been found that proper convergence of the coupled-channels calculations does not occur until 6-7 states in the rotational band have been included for even-nuclei, and approximately 12 for odd and odd-odd nuclei. Essentially all previous optical potentials are not properly converged because they did not use a sufficient number of coupled channels. This includes the recent high-quality potential of Soukhovitskii [13], which used five states, and we have found to be unconverged by approximately $20 \mathrm{mb}$ for low-energy incident neutrons. We note that these potentials have been empirically determined, so they generally describe the total cross section, but the separation of the contributions that go into the shape-elastic and absorption components is not accurately determined if the calculation is not adequately converged.

After we began developing our own potential, we became aware that the high-quality potential of Soukhovitskii et al. [13] is a significant improvement over prior actinide potentials, and almost fully converged. The parameterization of the energy dependence of the parameters in the Soukhovitskii potential is somewhat complicated, however, and a comprehensive understanding of the uncertainties in these parameters is more difficult to achieve in consequence. More importantly, it is beneficial to arrive at an independent potential to gain greater confidence in our findings. Indeed, throughout this work, our new optical potential was used for many comparisons with both experimental data and the evaluated nuclear data, and was compared with the Soukhovitskii potential. For the most part, our potnentail is $80 \%$ complete, needing some refinement. Our approach, which is quite different than that of Soukhovitskii is described in more detail in Appendix B. 


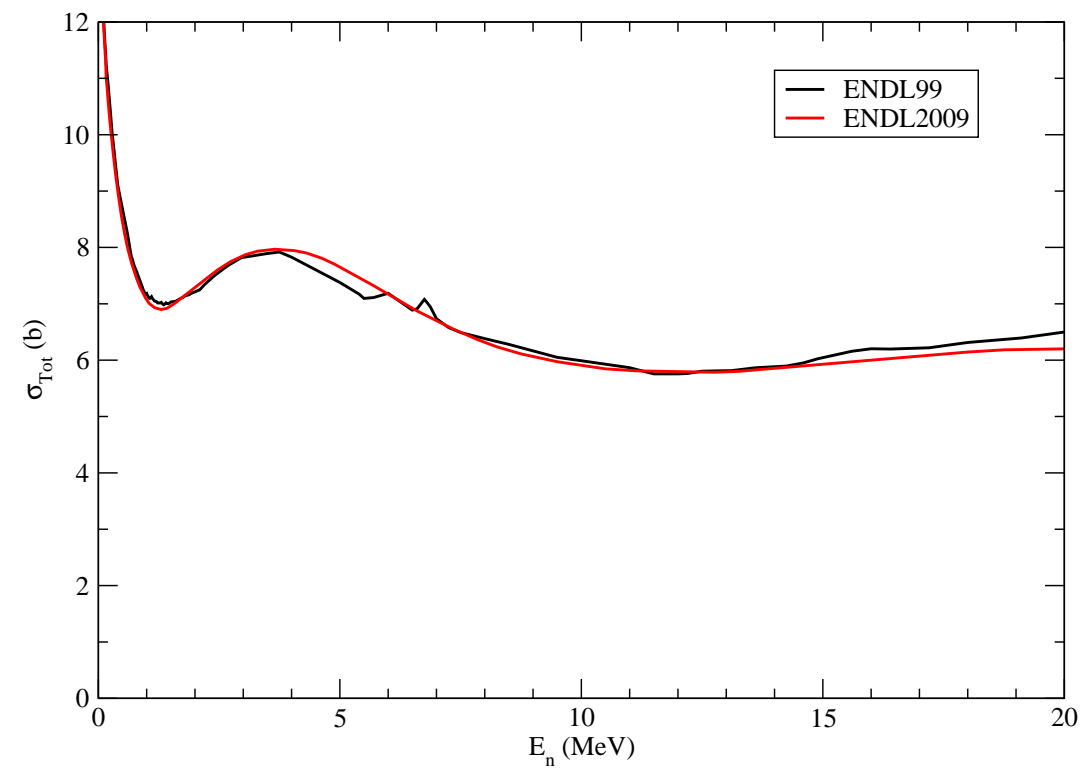

FIG. 2: Comparison between just the ENDL99 (black) and ENDL2009 (red) libraries for the total cross section.

\section{TOTAL CROSS SECTION}

In this section, we examine the total cross section in each library and compare them with experimental data as well as the results obtained from the two modern optical potentials Soukhovitskii [13] and our FLAP-A2.99d. Before showing comparisons with experimental data, we show in Fig. 2 a comparison between just the ENDL99 and ENDL2009 libraries for the total cross section on a linear plot for incident neutron energies, $E_{n}$ up to $20 \mathrm{MeV}$. The most striking feature is the structure exhibited in the ENDL99 library at $E_{n} \approx 5-7 \mathrm{MeV}$. These small peaks are unphysical, and are likely due to past attempts to update the library by modifying cross sections in other channels. It is likely that these modifications were made without explicitly refining the total cross section. Thus errors may be induced when the partial cross sections are summed up to make the total cross section.

A comparison between experimental data [3-9] with the ENDL99 and ENDL2009 libraries and the Sukhovitskii and FLAP-A2.99d potentials is shown in Figure 3. The experimental situation at lower energies is more complicated as this is where isolated resonances start to 


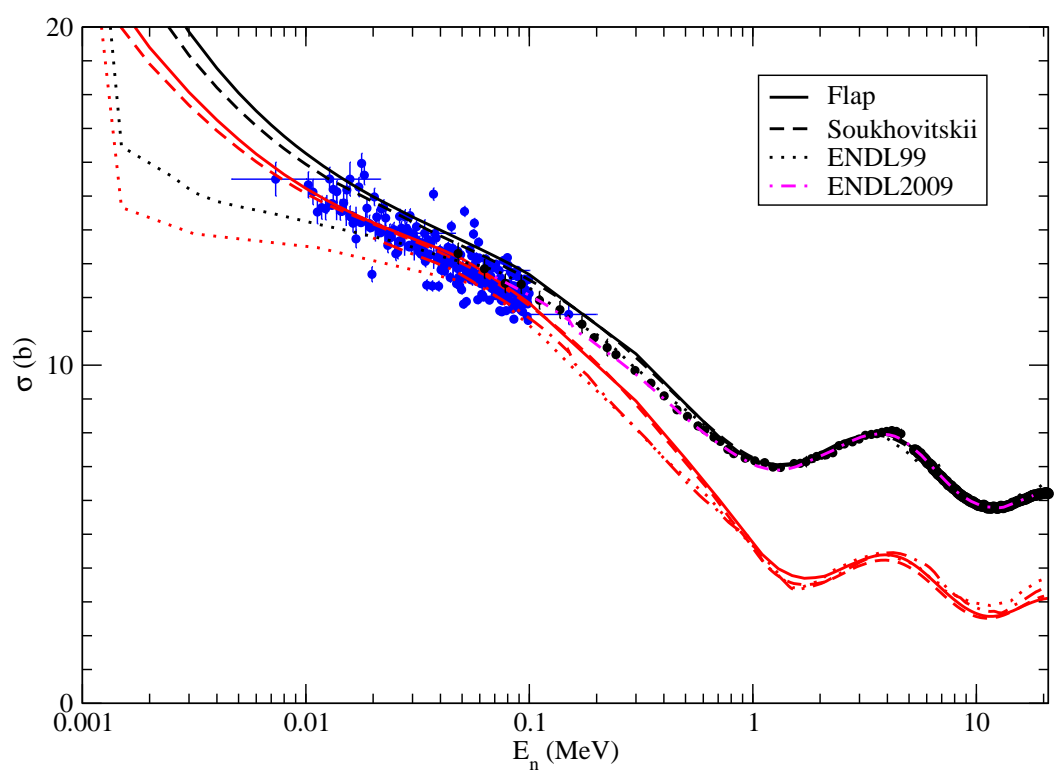

FIG. 3: Comparison of the total cross section and elastic cross section between ENDL99 (dotted), ENDL2009 (dot-dash), the Soukhovitskii [13] (dashed) and FLAP-A2.99d Solid) potentials, and high-quality experimental data (circles with error bars) [6-9] and other lower-quality experimental data [3-5] (blue circles with error bar). For all but ENDL2009, the total cross section is in black, while the elastic is in red. For ENDL2009, the total cross section is displayed in magenta in order to provide greater contrast.

enter, and the data [3-5] tends to fluctuate because of this. This fluctuating data is shown in the figure with blue circles and error bars. In the energy region $0.020 \leq E_{n} \leq 0.800$ MeV, both ENDL99 and END2009 give an overall good description of the experimental data. The two modern optical potentials slightly overestimate the measured total cross in this region. We found this behavior to be a consequence of increasing the number of coupled states to enhance convergence. We found that the total cross section increased slightly for the Soukhovitskii potential when the number of coupled channels was increased from five (the number used to fit the potential) to seven, where overall convergence is better. This is a difficult systematic issue that eventually needs to be resolved for improved optical potentials. The lines in the figure for ENDL2009 do not extend below $\approx 70 \mathrm{keV}$ as this is the region where individual resonances enter. At the lowest energies, the ENDL99 cross 
sections substantially undershoot ENDL2009 and the modern potentials until roughly $2 \mathrm{keV}$ where resonances dominate. As noted, these lower energies involve many resonances, and an averaging of some sort has to be performed. The data shown with blue circles (and error bars) is not averaged over as large an interval as the black circles. Still, these fluctuating lower energy data do not seem to support the smaller cross section exhibited by the ENDL99 library in the energy region $1 \leq E_{n} \leq 20 \mathrm{keV}$.

\section{ELASTIC ANGULAR DISTRIBUTIONS}

Elastic scattering is the process where the incident neutron interacts with the target nucleus without imparting any of its energy to the target, except through recoil. Thus, the target nucleus remains in the same initial state. Elastic processes are made up of two components. The first is scattering of the neutron from the external potential, namely the optical potential, and is referred to as "shape elastic". This component is generally forward-angle dominant, especially at higher incident energies, and is determined solely by the parameters of the potential. The second component arises when the incident particle is absorbed by

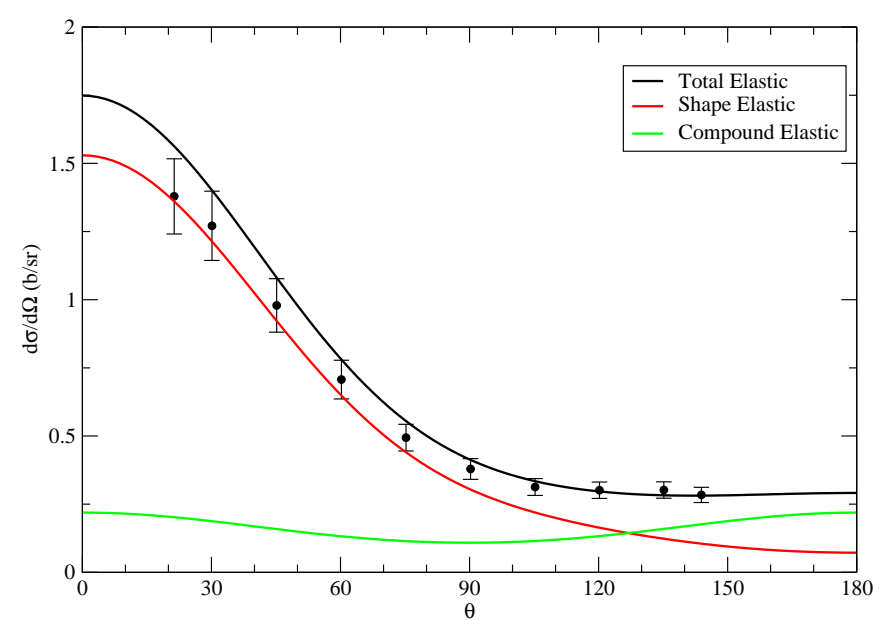

FIG. 4: Contributions of the shape elastic (red line) and compound elastic (green line) to the elastic-scattering angular distributions (black line) at $E_{n}=0.475 \mathrm{MeV}$. The calculation is compared with experimental data in Ref. [10]. 
the target and then re-emitted to the ground state. Generally, this emission comes from the compound nucleus, and is thus referred to as "compound elastic". Since it is emitted from the compound nucleus, this component is symmetric about $90^{\circ}$ in the scattering angle. It is, of course, not possible to distinguish between these two components experimentally. Thus the evaluations that utilize modeling combine these two (although theoretical calculations yield separate estimates for the two contributions). The compound elastic component contributes significantly at lower energies, typically $1 \mathrm{MeV}$ or less. This is because with increasing excitation energy, the neutron emitted from the compound nucleus can decay
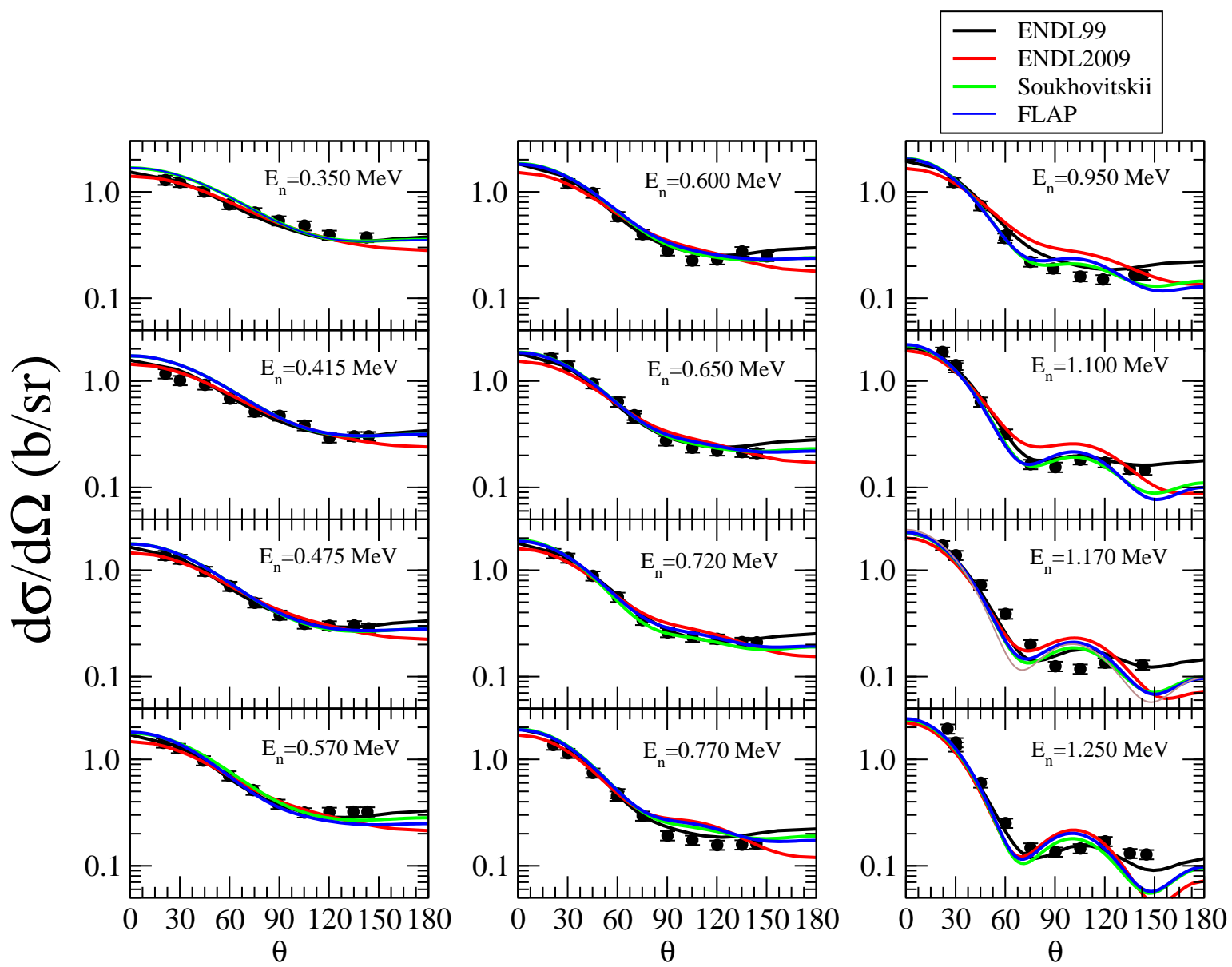

FIG. 5: Comparison between angular distributions from experimental data [10] (black circles with error bars) and the ENDL99 (black line), ENDL2009 (red line) libraries, and calculations with the Soukhovitskii (green line) and FLAP-A2.99d (blue) optical potentials. 
to other excited states (contributing to the inelastic, or $\left(\mathrm{n}, \mathrm{n}^{\prime}\right)$, channel). As more states become available for the neutron to decay to, the probability of decay back to the ground state decreases dramatically. The effect of the shape and compound elastic components can be seen in Figure 4 for $E_{n}=0.475 \mathrm{MeV}$. Since the shape elastic angular distribution is highly forward peaked, the compound elastic primarily affects backward scattering, and can have a significant contribution at these low energies.

The elastic scattering cross section and angular distributions in ENDL99 and ENDL2009 differ significantly. The best experimental data can test these libraries, and the results obtained from the two modern optical potentials Soukhovitskii and FLAP-A2.99d are now compared with the data of Smith [10] and Haouat et al. [11]. We show in Figure 5 the comparison between the angular distributions from experimental data [10] (black circles with
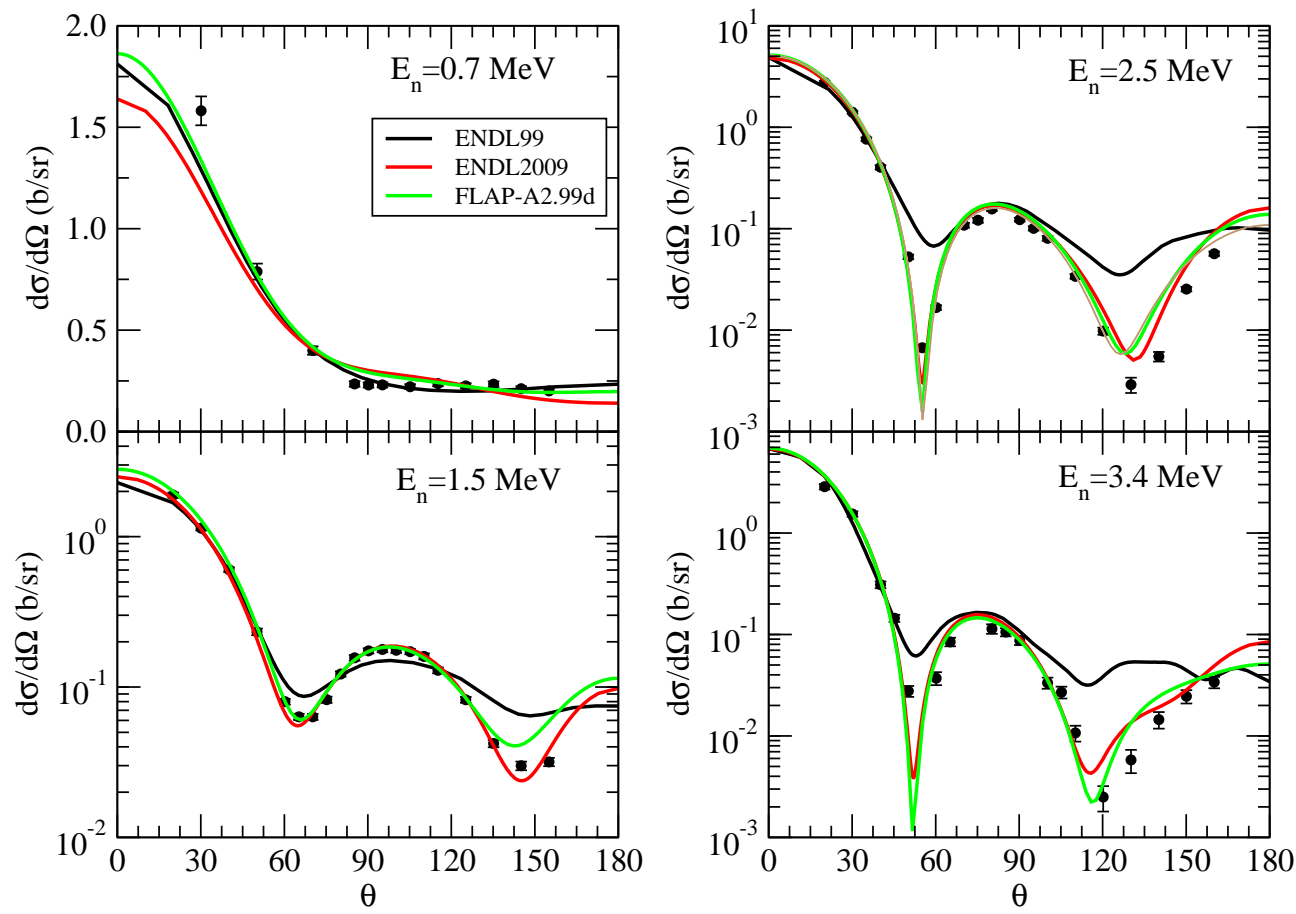

FIG. 6: Comparison of the differential elastic scattering cross section between experimental data [11] (black circles with error bars) and the ENDL99 (black line), ENDL2009 (red line) libraries, and calculations with the FLAP-A2.99d (green) optical potential. 
error bars) and the ENDL99 (black line), ENDL2009 (red line) libraries, and calculations with the Soukhovitskii (green line) and FLAP-A2.99d (blue) optical potentials. For ENDL99 and ENDL2009, the angular distributions were obtained using PYTHON routines written by Bret Beck to interpolate the tabulated data to the energies corresponding to experiment. In general, both libraries and the modern optical potentials give an overall good representation of the data. ENDL99, however, appears to be better at backward angles in the energy region for incident neutron energy, $E_{n}, 0.7-1.25 \mathrm{MeV}$. Of them all, ENDL2009 gives the least satisfactory representation of the data in this energy regime.

At higher energies, we turn to the data of Haouat et al. [11], which is shown in Figure 6 where the experimental differential cross section $d \sigma / d \Omega$ at $E_{n}=0.7,1.5,2.5$, and $3.4 \mathrm{MeV}$ is compared with ENDL99, ENDL2009, and the FLAP-A2.99d potential. At $E_{n}=0.7 \mathrm{MeV}$, the comparison with the Haouat data is similar as with the Smith data; ENDL99 is in slightly better agreement with experiment. On the other hand, at the higher energies, ENDL99 clearly misses the data, and ENDL2009 and FLAP-A2.99d are definitely superior in quality.

In addition to these angular distributions, Smith et al. [10] also constructed the decomposition of the angular distributions in terms of Legendre polynomials. The differential cross section may be written as

$$
\frac{d \sigma}{d \Omega}=\sum a_{L} P_{L}(\cos \theta)
$$

The coefficients $a_{L}$ are shown in Figure 7 for the ENDL99 and ENDL2009 libraries and the FLAP-A2.99d potential (the difference between FLAP-A2.99d and Soukhovitskii is minimal). The ENDL99 and ENDL2009 coefficients were obtained by first extracting angular distributions from the tabulated data using the interpolation routines of Beck and fitting the corresponding angular distributions to the Legendre expansion using the program MATHEMATICA. Note that the coefficient $a_{0}$ is the total elastic cross section divided by $4 \pi$. Overall, both evaluations and the FLAP calculation give comparable agreement with data, although the FLAP $a_{1}$ coefficient seems to rise and be too large for $0.1 \leq E_{n} \leq 0.5 \mathrm{MeV}$. The primary differences seen in the $a_{L}$ coefficients is that ENDL99 has a slightly higher value for $a_{2}$ for much of the energy range, and a slight decrease in $a_{4}$ for $E_{n}>1 \mathrm{MeV}$. These are the likely origin of the angular distributions in ENDL99 having less structure at backward angles for $0.7 \leq E_{n} \leq 1.3 \mathrm{MeV}$ seen in Figures 5 and 6 .

Finally, in Fig. 8 we compare the normalized angular distributions (scattering probabil- 

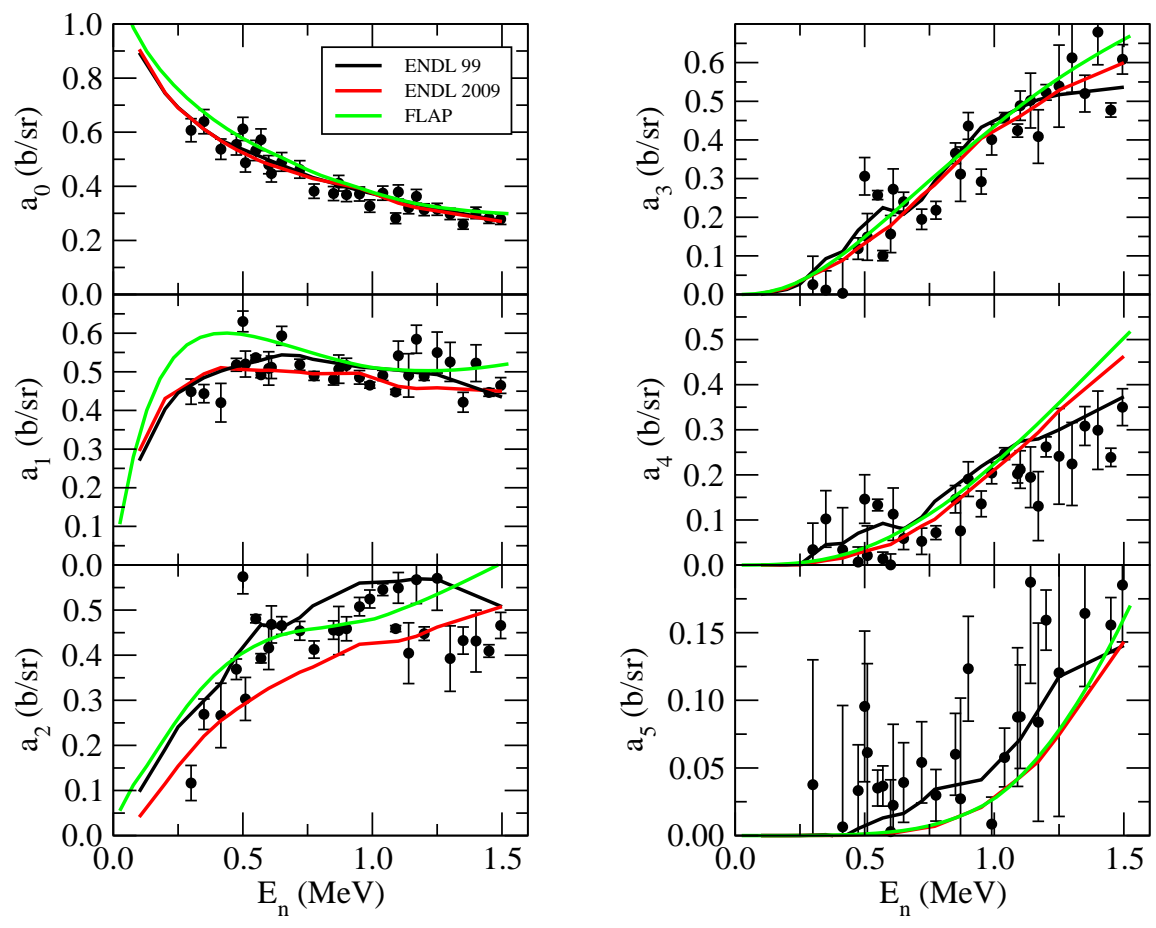

FIG. 7: Comparison of the Legendre coefficients in elastic scattering between experimental data [10] (black circles with error bars) and the ENDL99 (black line), ENDL2009 (red line) libraries, and calculations with the FLAP-A2.99d (green) optical potential.

ities $P(\mu)$ as a function of $\mu=\cos \theta$ normalized so that $\left.\int_{-1}^{+1} P(\mu) d \mu=1\right)$ in the ENDL99 and ENDL2009 libraries and the calculation with the FLAP-A2.99d potential for eight different incident neutron energies $E_{n}$. The main feature is that the ENDL99 library is more isotropic, i.e., less forward scattering, than either ENDL2009 or FLAP-A2.99d for energies above $1 \mathrm{MeV}$. In neutron transport applications, this would lead to more backward scattering.

The overall conclusion is that for energies below $E_{n}<1.5 \mathrm{MeV}$, the ENDL99 evaluation for the angular distributions appears to be slightly better than ENDL2009 or the calculations from FLAP-A2.99d (especially in the region $0.7 \leq E_{n} \leq 1.25 \mathrm{MeV}$. At higher energies, however, ENDL2009 and FLAP-A2.99d are clearly in much better agreement with data. The primary distinction between ENDL99 and ENDL2009 is that for incident neutron energies 

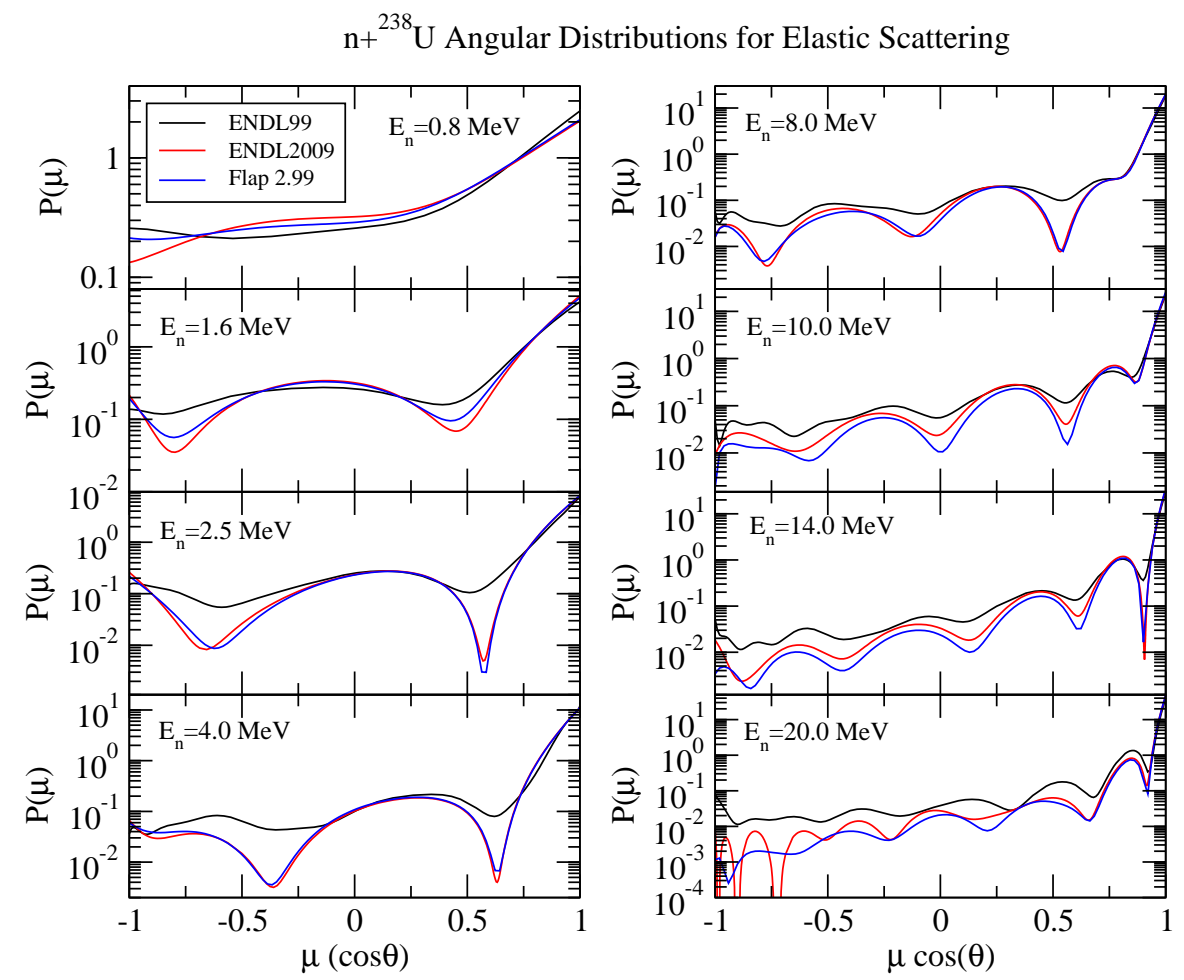

FIG. 8: Comparison between normalized angular distributions (normalized scattering probabilities as a function of $\mu=\cos \theta$ ) in the ENDL99 (black line), ENDL2009 (red line) libraries, and calculations with the FLAP-A2.99d (blue) optical potentials.

above $1 \mathrm{MeV}$, the elastic scattering in ENDL99 is less forward peaked, and, consequently, predicts greater backscattering.

\section{INELASTIC SCATTERING}

Inelastic scattering, or $\left(\mathrm{n}, \mathrm{n}^{\prime}\right)$, occurs when the incident neutron interacts with target nucleus, and transfers some of its energy to promote the target into an excited state. Similar to elastic scattering, inelastic processes are comprised of multiple components. The first is a "direct" interaction between the incident particle and the target, where an excited state is populated without the particle being absorbed. This is accounted for with the optical potential by solving a series of coupled-channels equations. This is important for deformed 
nuclei, where the direct process can excite states in the ground-state rotational band through quadrupole and hexadecupole excitations. The second component, which itself is made of two sub-components, occurs when the incident neutron is absorbed by the target, and the neutron is emitted with less energy, leaving the target in an excited state. The contributions occurring after absorption are: 1) the neutron is emitted from the compound nucleus, and 2) the neutron is emitted prior to complete formation of the compound nucleus, i.e., before the neutron interacts sufficiently to share all its energy within the nucleus, and thus lose memory of the initial conditions. This last component is the so-called "pre-equilibrium" emission. Neutrons emitted from the compound nucleus tend to be lower in energy (roughly Maxwellian peaked at $1 \mathrm{MeV}$ ) than from the equilibrium process, and are also symmetric about $90^{\circ}$. On the other hand, pre-equilibrium neutrons have a higher energy, and tend to be more forward peaked, having an angular dependence reasonably approximated [21] by

$$
\frac{d \sigma}{d \Omega} \approx \frac{a}{\sinh (a)} \exp (a \cos \theta)
$$

where $a$ is determined by the incident and emitted energies, as well as the target charge and mass.

The treatment of inelastic scattering is somewhat different in the ENDL99 and ENDL2009 libraries. The inelastic process involves an excitation to a discrete state in the target nucleus. Several discrete states have been identified experimentally, and listed in the Table of Isotopes or in the ENSDF evaluations. It is clear, however, that for all nuclei these compilations of states are not complete above some energy. Consequently, evaluations based on modeling with Hauser-Feshbach usually consider a set of discrete states assumed to be complete up to an energy $E_{c u t}$, and then use a continuous level density to describe states above $E_{c u t}$. This abrupt transition from discrete to continuous was used in both ENDL99 (and so far in the calculations performed by us) and ENDL2009. In ENDL2009, however, there is an additional treatment of "discrete" states at energies well above $E_{c u t}$. This treatment is based on the observation [22] that there is an excess of cross section for ( $\left.\mathrm{n}, \mathrm{n}^{\prime}\right)$ reactions populating lower energies $(\leq 4 \mathrm{MeV})$ than predicted by Hauser-Feshbach and pre-equilibrium models. This excess of strength is not observed going to any specific known states. Indeed, the density of states in this region is quite high, and it would be difficult to resolve transitions to states in this excitation energy region. In an attempt to properly account for this excess strength, the ENDF/B-VII.0 evaluation (on which ENDL2009 was based) was performed by 
adding eighteen fictitious states to ${ }^{238} \mathrm{U}$ with angular momentum $J^{\pi}=2^{+}, 3^{-}$, and $4^{+}$and excitation energies between 1.17 and $3.909 \mathrm{MeV}$ and performing a calculation within the distorted wave Born approximation (DWBA) to estimate the direct inelastic scattering to these states. These additional states are, in effect, embedded into the continuous states used in the Hauser-Feshbach treatment. It should pointed out that these additional states are not representative of actual levels observed in ${ }^{238} \mathrm{U}$, but, instead, were added in order to account for a discrepancy observed between theoretical calculations within the Hauser-Feshbach (plus pre-equilibrium) formalism and experimental data. The presence of these "states" does point to limitations in our overall understanding of the physics processes occurring. The overall

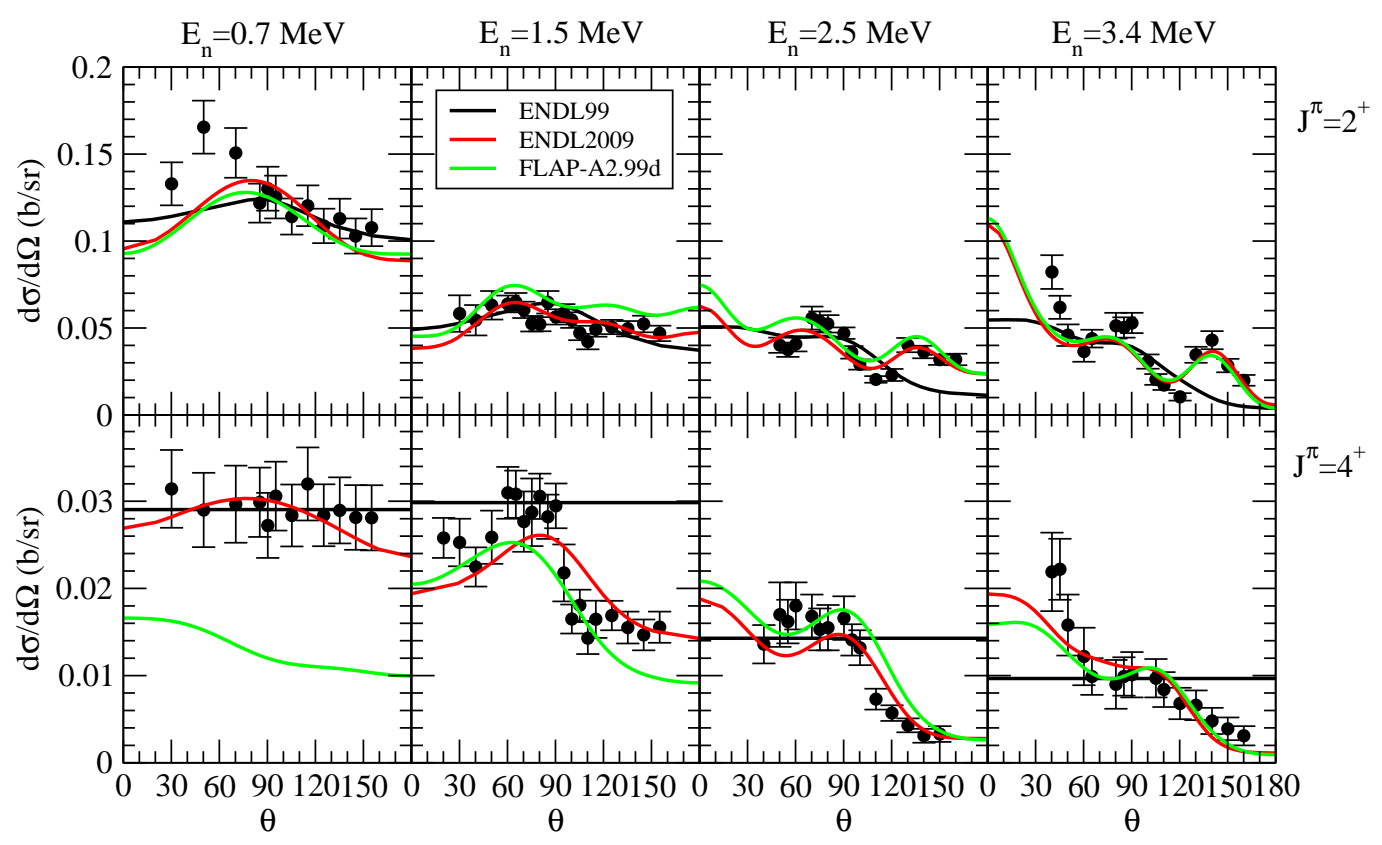

FIG. 9: Comparison of the differential inelastic scattering cross section between experimental data [11] (black circles with error bars) and the ENDL99 (black line), ENDL2009 (red line) libraries, and calculations with the FLAP-A2.99d (green) optical potential for excitations to the lowest rotational $J^{\pi}=2^{+}$and $4^{+}$states at incident energies $E_{n}=0.7,1.5,2.5$, and $3.4 \mathrm{MeV}$. The upper four graphs are for the $2^{+}$final state, and the lower four are for the $4^{+}$. 
strength going to these states is relatively small, of the order 5-20 mb (in their peak at roughly $E_{n} \sim 6 \mathrm{MeV}$ ) for any of the states. Generally, their sum, contributing to all eighteen states, amounts to about $150 \mathrm{mb}$ at the peak, and slowly decreases with increasing $E_{n}$. In comparison to the absorption cross section $(\approx 3 \mathrm{~b})$, these channels are relatively small. But at incident neutron energies of roughly 7-10 MeV, they make up a considerable fraction of the total (n,n') cross section. In the ENDF/B-VII.0 report [1], it is also indicated that the addition of these states improves calculations with critical assemblies.

\section{VII.1. Inelastic scattering to specified states below $E_{c u t}$}

Inelastic scattering to discrete states in ${ }^{238} \mathrm{U}$ has the two components as described above. The first is a direct reaction to the state, while the second arises from emission of a neutron following the absorption of the projectile (both compound and pre-equilibrium). The direct reactions are accounted for by performing a coupled-channels calculation with the optical potential, while the absorption component in computed with the Hauser-Feshbach code (including a pre-equilibrium model). The coupled channels calculation involves excitations to states within the ground-state rotational band. In ENDF/B-VII.0, the coupled channels calculations were performed with three states within the rotational band. As noted earlier, this was later found not to be sufficient to attain convergence in the coupled-channels treatment. Nevertheless, the optical parameters used in ENDF/B-VII were tuned to describe the total cross section. Our optical model was determined using 6 or 7 states in the rotational band, which gave excellent convergence.

In Figure 9, the differential cross section, $d \sigma / d \Omega$, is shown for excitations to the $J^{\pi}=2^{+}$ and $4^{+}$states in the ground-state band for the ENDL99 and ENDL2009 libraries, as well as a Hauser-Feshbach calculation using the FLAP-A2.99d potential. These are compared with experimental data [11] for incident neutron energies of $0.7,1.5,2.5$, and $3.4 \mathrm{MeV}$. Generally speaking, both libraries and the calculation give a reasonable representation of the cross section for the $2^{+}$state, although ENDL99 starts to show significant deficiencies at $E_{n}=3.4 \mathrm{MeV}$. For the $4^{+}$state, the ENDL99 library is isotropic, which disagrees with the experimental data. In this case, ENDL2009 is superior. It is interesting to note that the FLAP-A2.99d results are in agreement with the data except at $E_{n}=0.7 \mathrm{MeV}$. The fact that the experiment is more isotropic at this energy indicates that the compound component 

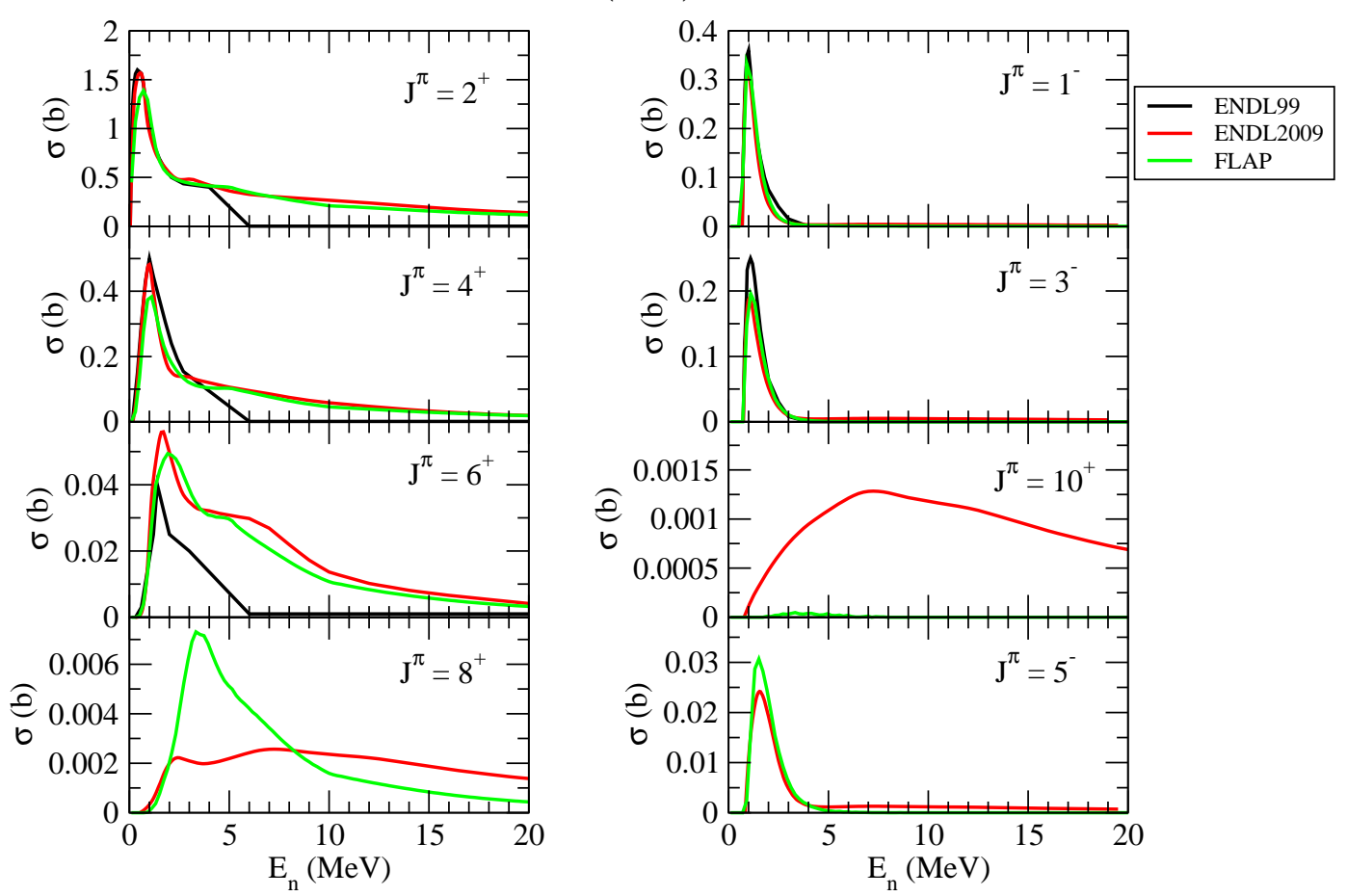

FIG. 10: Comparison of the total $\left(\mathrm{n}, \mathrm{n}^{\prime}\right)$ cross section to excited states in ${ }^{238} \mathrm{U}$ labelled by their angular momentum as a function of incident neutron energy $E_{n}$.

is important. This is not reproduced in the calculation. By analyzing the transmission coefficients leading to various angular momenta in the compound nucleus, the fact that calculation significantly underpredicts excitation to the $4^{+}$state is an indication that opticalpotential parameters might need to be refined in order to increase the $l=3$ transmission coefficient at low incident energies, or that there is a problem with the experimental data for this case. We note that the Soukhovitskii potential as well as the potential described in [11] show a similar underprediction. This issue is not understood at present.

In Figure 10, the total inelastic $\left(\mathrm{n}, \mathrm{n}^{\prime}\right)$ cross section is shown for the ENDL99 and ENDL2009 libraries and the FLAP-A2.99d results for excitations to the lowest eight states in ${ }^{238} \mathrm{U}$. There is some agreement between the three evaluations at low energies $(<3 \mathrm{MeV})$ for several states. For the $2^{+}, 4^{+}$, and $6^{+}$states, the curves show a typical pattern with a fairly sharp peak at the lowest energies, followed by a slow decline. The "sharp" peak 
is indicative of compound (quite visible in the panels for the $1^{-}, 3^{-}$, and $5^{-}$states), while the slow decline arises from the direct component. The sharp decline is due to the rapid opening of additional excited states as the incident energy is increased. In effect, there is a significant increase in the competition between states, and thus the likelihood for the decay to occur to one given state decreases. Reasonable agreement is exhibited between the three evaluations for incident energies below $3 \mathrm{MeV}$. ENDL99, however, displays a sharp decline, going to $1 \mathrm{mb}$ at $E_{n}=6 \mathrm{MeV}$, and later remains constant until $20 \mathrm{MeV}$. This is unphysical. This is not to say that ENDL99 underestimates the total $\left(\mathrm{n}, \mathrm{n}^{\prime}\right)$ cross section, as it is possible that these excitations are eventually collected into the component exciting the continuous levels. However, there is a significant difference in the angular distributions between

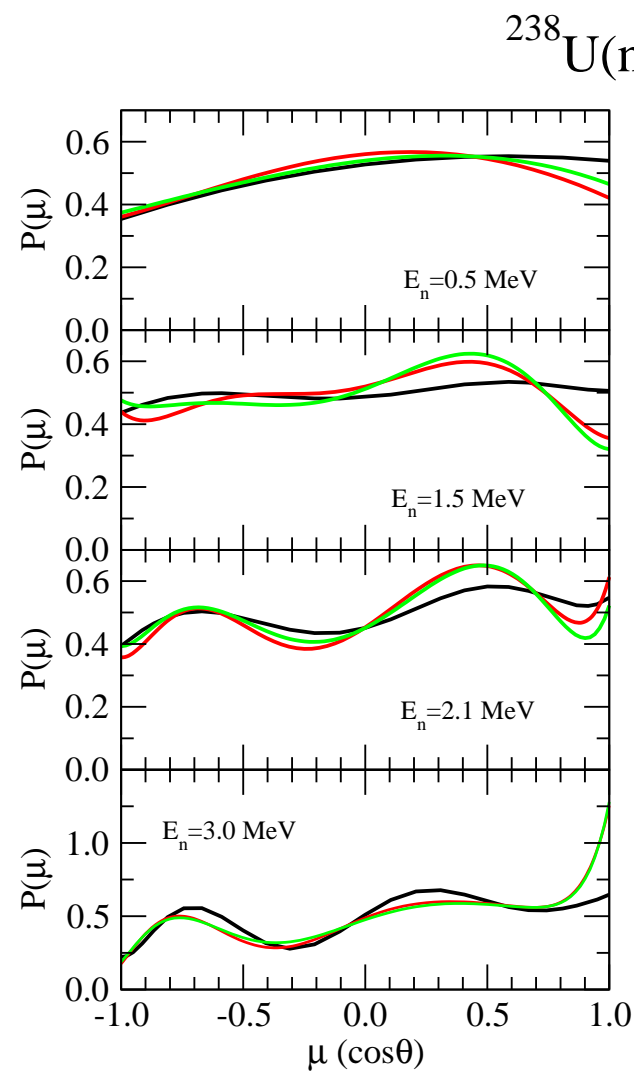

$\left.\mathrm{n}^{\prime}\right) ; \mathrm{J}=2^{+}$

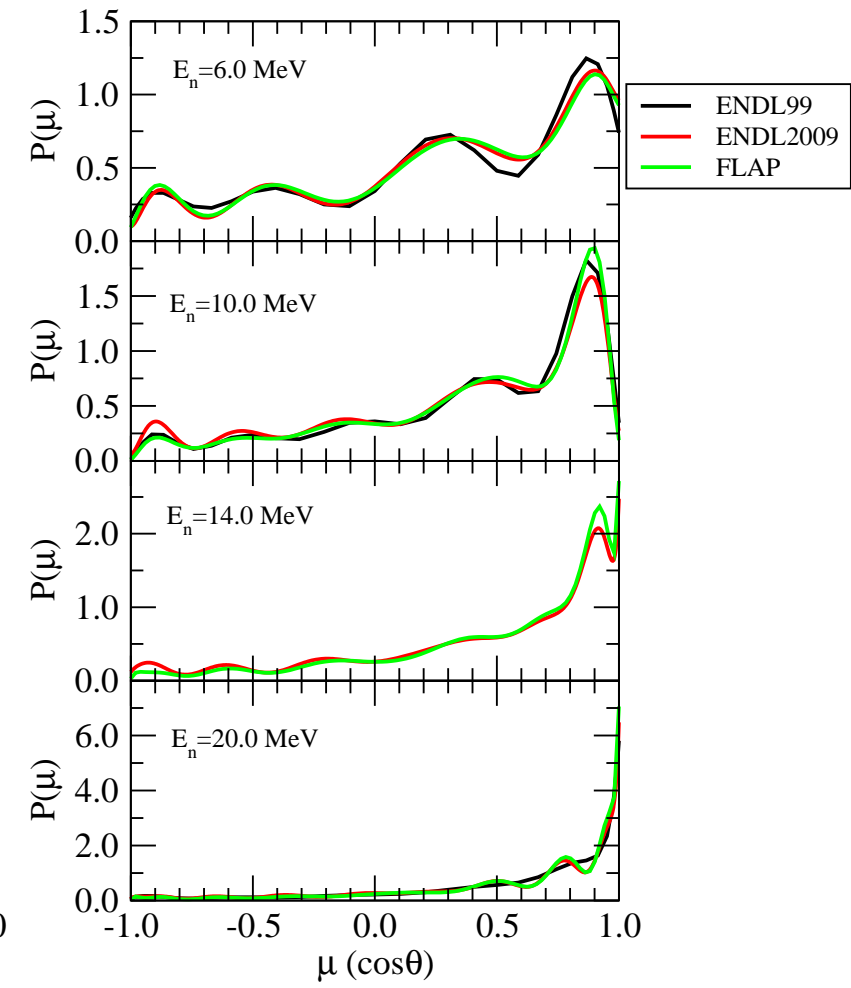

FIG. 11: Comparison of the normalized angular distributions in the ENDL99 (black) and ENDL2009 (red) libraries and obtained with the FLAP-A2.99d optical potential for $\left(\mathrm{n}, \mathrm{n}^{\prime}\right)$ reactions to the $J^{\pi}=2^{+}$state in ${ }^{238} \mathrm{U}$ at various incident neutron energies. 
compound emission and a direct reaction. Compound emission tends to be approximately isotropic (in ENDL99 reactions to unspecified states in the continuous spectrum are completely isotropic), while the direct reaction is more forward peaked (this is well illustrated in Fig. 11). Overall, ENDL2009 and FLAP-A2.99d cross sections are in general agreement except for the $J^{\pi}=8^{+}$and $10^{+}$states. The structure of these cross sections indicates that they were estimated with a DWBA calculation, rather than a coupled-channels calculation, which is likely not as accurate. However, we note that the cross section to these states is fairly small.

In Figure 11, the scattering probability (as a function of $\mu=\cos \theta$ ) is shown for excitations to the $J^{\pi}=2^{+}$state for eight different incident neutron energies for the ENDL99 and ENDL2009 libraries and the FLAP-A2.99d calculation. Overall, there is reasonable agreement between all three (note ENDL99 is not shown at $E_{n}=14 \mathrm{MeV}$ as there is no entry at this energy, and the data were not interpolated for this plot), but on the whole ENDL99 is more isotropic. ENDL2009 and FLAP-A2.99d are in excellent agreement. While the ENDL99 angular distributions are similar to those in ENDL2009, it is important to note that the ENDL99 cross section to this state drops to $1 \mathrm{mb}$ at $E_{n}=6.0 \mathrm{MeV}$, and is constant at $1 \mathrm{mb}$ to $20 \mathrm{MeV}$. Thus, even though the library contains a quite reasonable normalized scattering probability, it is rarely accessed because the magnitude of the cross section in the library is unphysically too small. Given the overall importance of the $2^{+}$state (the cross section is about $50 \mathrm{mb}$ for $E_{n} \geq 3 \mathrm{MeV}$ ), the overall effect is that inelastic scattering in ENDL99 will be a bit more isotropic, while in ENDL2009, it will be a bit more forward peaked.

Lastly, in ENDL99 the angular distributions for excitations to states above the $44 \mathrm{keV}$ $J^{\pi}=2^{+}$state are all isotropic. As seen in Figure 9, this is unphysical for the states excited by direct mechanisms, i.e., the $J^{\pi}=2^{+}-8^{+}$states in the ground-state band. For the other states, these are primarily populated via the compound nucleus, which is nearly isotropic, and symmetric about $90^{\circ}$. For $E_{n}>10 \mathrm{MeV}$, pre-equilibrium emission becomes more important, which is more forward peaked. However, as seen in Figure 10, the cross section to these states is fairly small at these energies. 


\section{VII.2. Inelastic scattering to specified states above $E_{c u t}$}

As mentioned above, there is an observed excess of cross section in the experimental data of Ref. [22] relative to expectations from model calculations. While fairly small, it is indicative of the limitations in current theoretical approaches, and shows that some corrections need to be applied. This excess was accounted for in ENDL2009 by introducing a set of 18 levels above the energy cutoff $E_{c u t}$ and performing a DWBA calculation with the coupled-channels optical model code ECIS. These levels do not correspond to any known levels in ${ }^{238} \mathrm{U}$ and were introduced as an "engineering" approach to account for the excess cross section. Shown in Fig. 12 are the four components making up the inelastic cross sec-

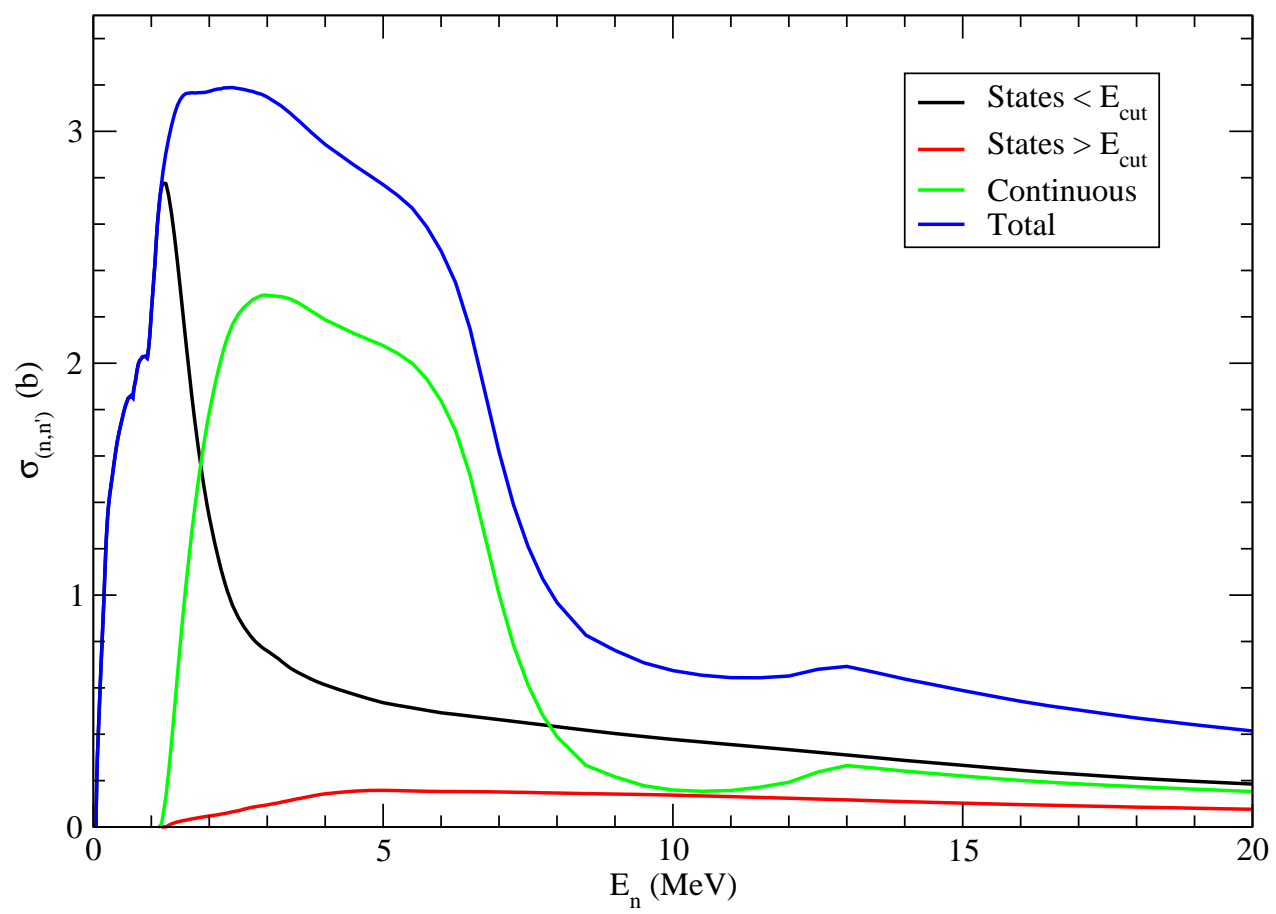

FIG. 12: Comparison of the components making up the inelastic cross section in ENDL2009 as a function of incident neutron energy, $E_{n}$. The total is given by the blue line, while contributions to specified states below $E_{\text {cut }}$ are in black, and those to "specified" states above $E_{\text {cut }}$ are in red. The contribution arising from unspecified states described by a continuous level density is in green. 
tion in ENDL2009. The sum of the cross section going to all unspecified states above the cutoff is shown in red. This peaks at about $150 \mathrm{mb}$ at $E_{n} \approx 5 \mathrm{MeV}$ and exhibits a slow decrease in cross section with increasing $E_{n}$. This is typical of "direct-like" reactions, and is different from compound, which tend to decrease more quickly after the incident energy is much greater than the energy of the state. In addition, the angular distributions for these states tends to start mostly isotropic with a slight forward directionality at lower energies near the threshold, and become forward focused with increasing incident energy as shown in Fig. 13 for transitions to two (fictitious) states with energy 1.440 and $2.940 \mathrm{MeV}$.

We also note the "kink" in the component to unspecified states above $E_{\text {cut }}$ at $E_{n} \approx$ $12-13 \mathrm{MeV}$. This is a curious feature, and in all likelihood is unphysical.

\section{VII.3. Inelastic scattering to unspecified states}

As mentioned earlier, evaluations explicitly treat a number of discrete states up to a welldefined excitation energy. Above this, the discrete states are replaced with a continuous level density. This type of treatment is required even though there are known levels above this

\section{$\left(n, n^{\prime}\right)$ to state at $1.4400 \mathrm{MeV}$}

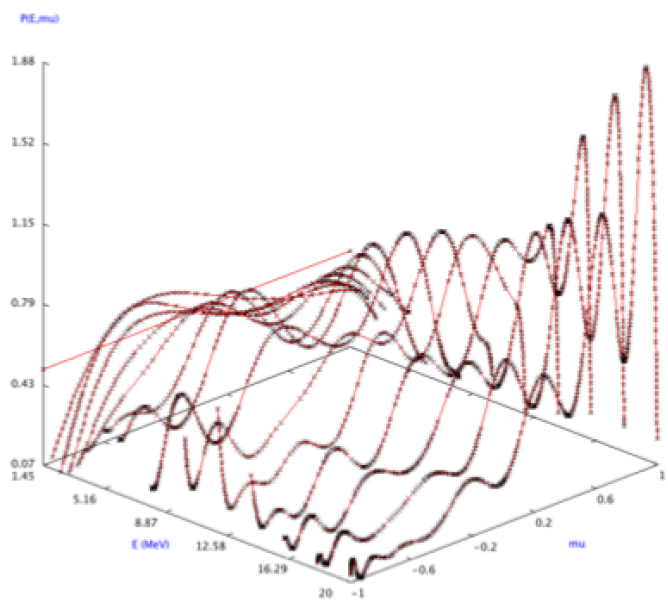

(n.n') to state at $2.940 \mathrm{MeV}$

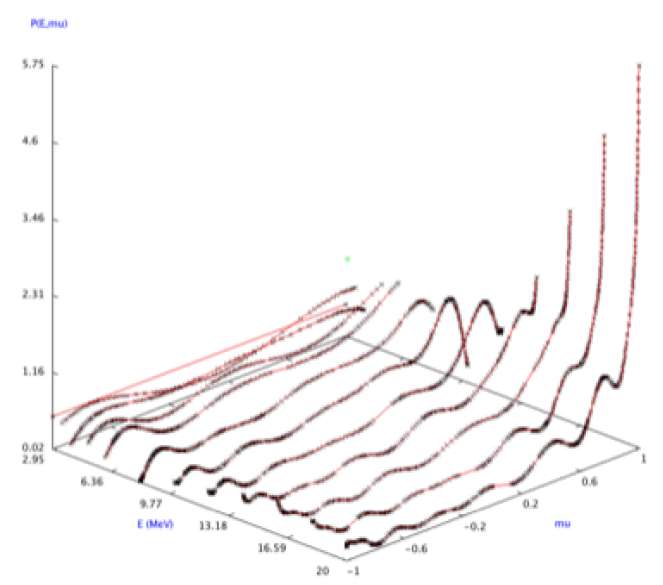

FIG. 13: Angular distributions for transitions to two "specified" states above $E_{c u t}$ with excitation energies at 1.440 and $2.940 \mathrm{MeV}$. The incident neutron energy is on the left-bottom axis, with increasing energy to the front. The front-right axis is $\cos \theta$ with the forward direction towards the right. 
cutoff. In practice the density of states becomes too large for experiments to resolve them, and in plots of the cumulative level density it becomes abundantly clear that the known levels are not complete because the cumulative density tends to flatten out, rather than continue to increase exponentially, which is the physically correct behavior. The density of states is typically modeled via the standard Gilbert and Cameron approach, where an exponentially increasing level density is used at low to moderate excitation energies, which then transitions to a Fermi-gas picture. The parameters for the level density are fixed by

${ }^{238} \mathrm{U}\left(\mathrm{n}, \mathrm{n}^{\prime}\right)$ to Continuous levels
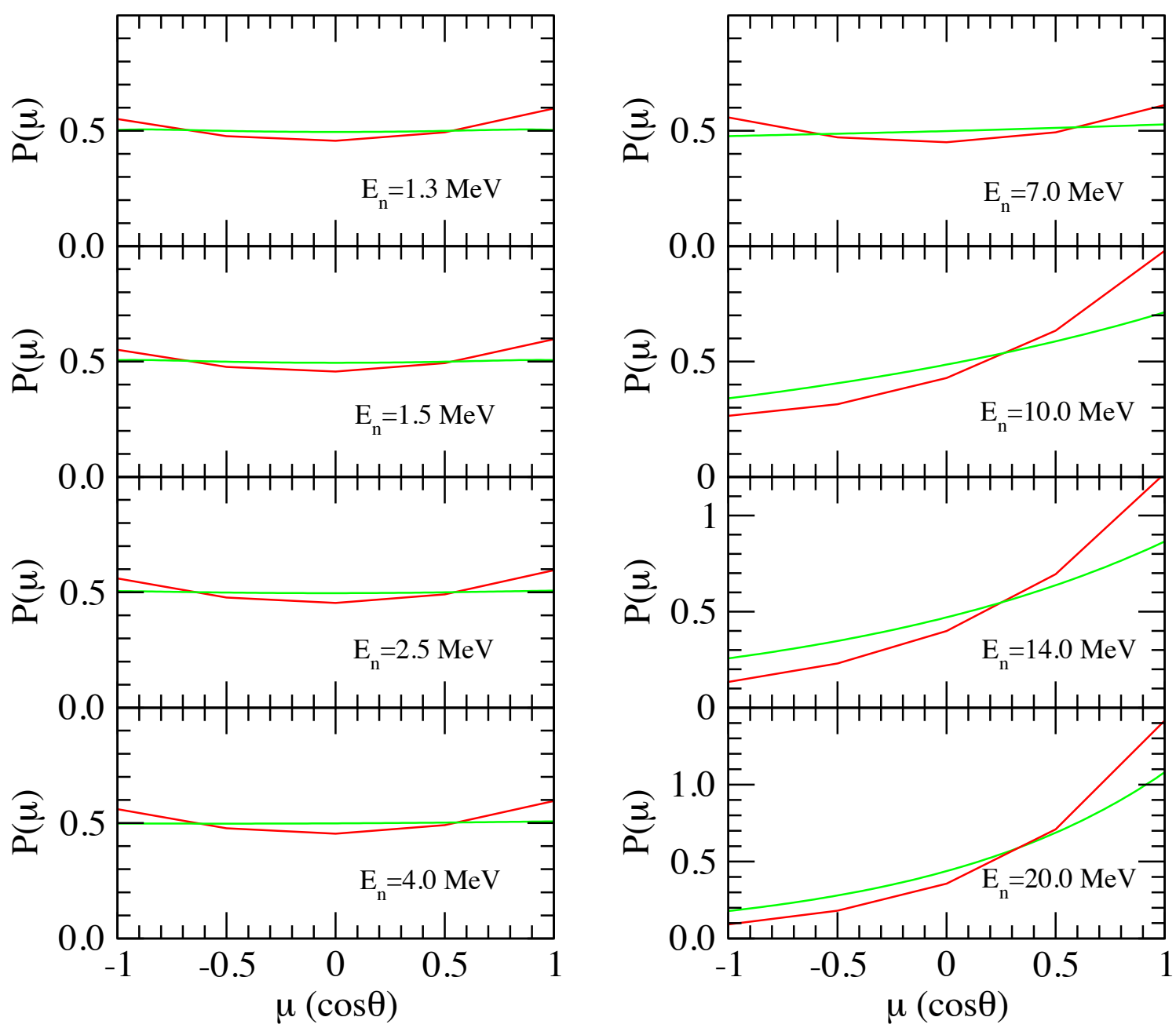

FIG. 14: Comparison of the normalized angular distributions in ENDL2009 (red curves) and our modeled results with the FLAP-A2.99d potential (green curves) for inelastic scattering to unspecified states above $E_{c u t}$ for eight incident neutron energies. 
reproducing the measured level spacing of $S$-wave resonances at the neutron separation energy and matching the cumulative density of discrete states at low excitation energy. The contribution arising from these states in the ENDL2009 library is shown by the green line in Fig. 12. At incident neutron energies greater than 2-3 MeV, transitions to unspecified states dominate the $\left(\mathrm{n}, \mathrm{n}^{\prime}\right)$ cross section. In the ENDL99 library, the angular distribution to these states above $E_{\text {cut }}$ is isotropic. In ENDL2009, they are more isotropic at the lowest incident energies, but become more forward peaked at higher incident energies due to the increased influence of pre-equilibrium emission. This is shown in Fig. 14, where ENDL2009 (red curves) is compared with our modeled results using the FLAP-A2.99d optical potential (green curves).

In addition to the angular distributions, the emitted neutron energy must also be specified. While examining the outgoing neutron spectrum from ENDF/B-VII.0 and ENDL2009,

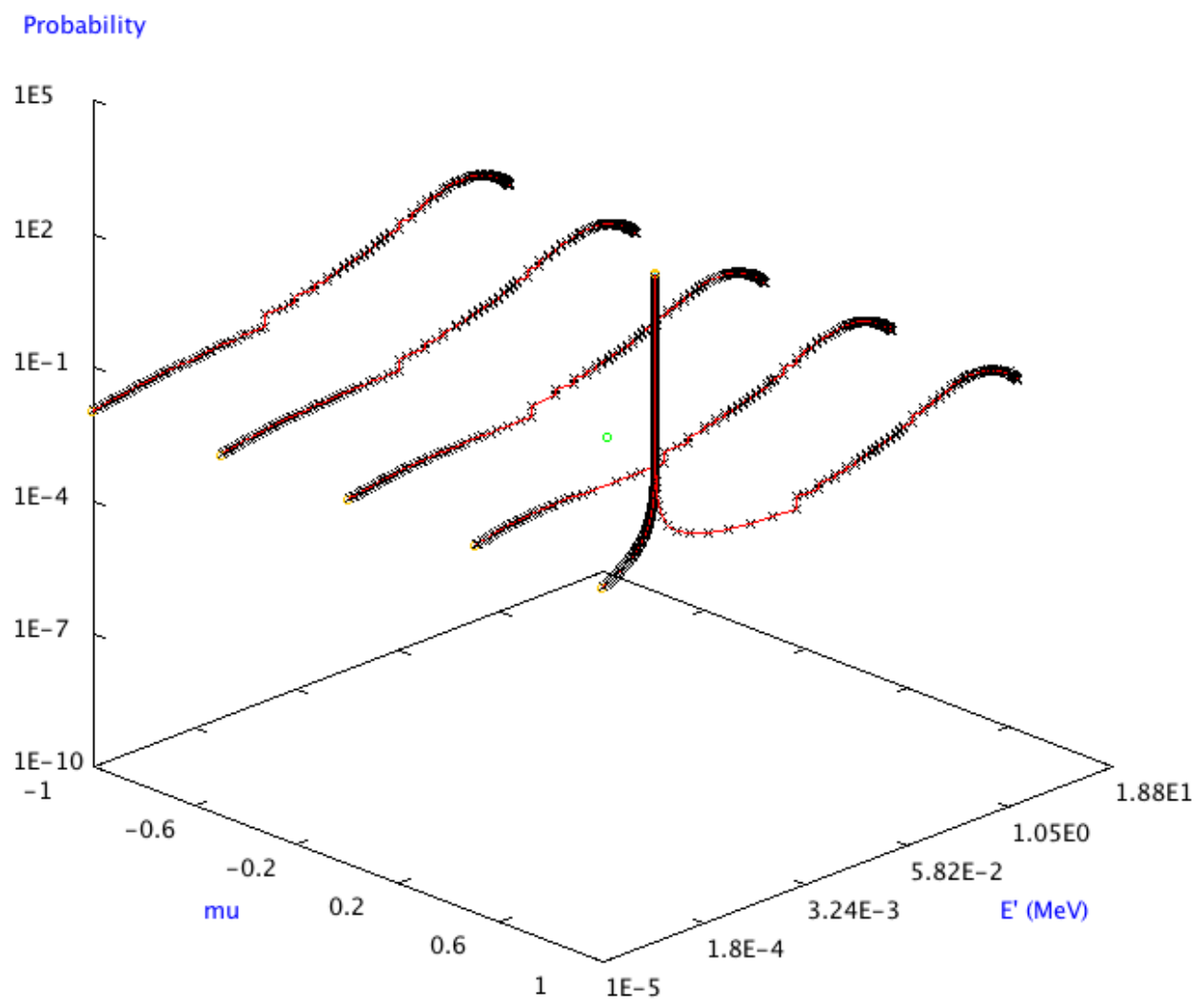

FIG. 15: Emission spectrum for transitions to unspecified states in the ENDL2009 library for various outgoing angles $(\mu=\cos \theta)$ 
we noticed an odd feature in the forward direction. This is shown in Fig. 15 where the emission probability is shown for outgoing angles $(\mu=\cos \theta)$ and outgoing energies for incident neutron energy of $1.9 \mathrm{MeV}$. The odd feature is seen at very low energies $\approx 10^{-4} \mathrm{MeV}$ in the forward direction $(\mu=1)$, where there is the appearance of a very narrow resonance structure. This feature is present at essentially all the listed incident neutron energies in the ENDL2009 library and is entirely unphysical. It is due to the way the data is parameterized and then reconstructed in the ENDF/B-VII.0 library. A more detailed picture of the emission probability is shown in Fig. 16 for incident neutron energies of $2.5 \mathrm{MeV}$. There is a sharp peak near neutron emission energies of $4 \times 10^{-5} \mathrm{MeV}$. The red curve shows the cumulative probability, and shows that while the unphysical peak is extremely high it only mildly skews the probability of the emission spectrum, with $\approx 10^{-4}$ probability.

\section{CRITICAL ASSEMBLIES}

In addition to comparing the libraries against each other and experimental data, we can also test the quality of nuclear data, in an integral way, via simulations for critical assemblies

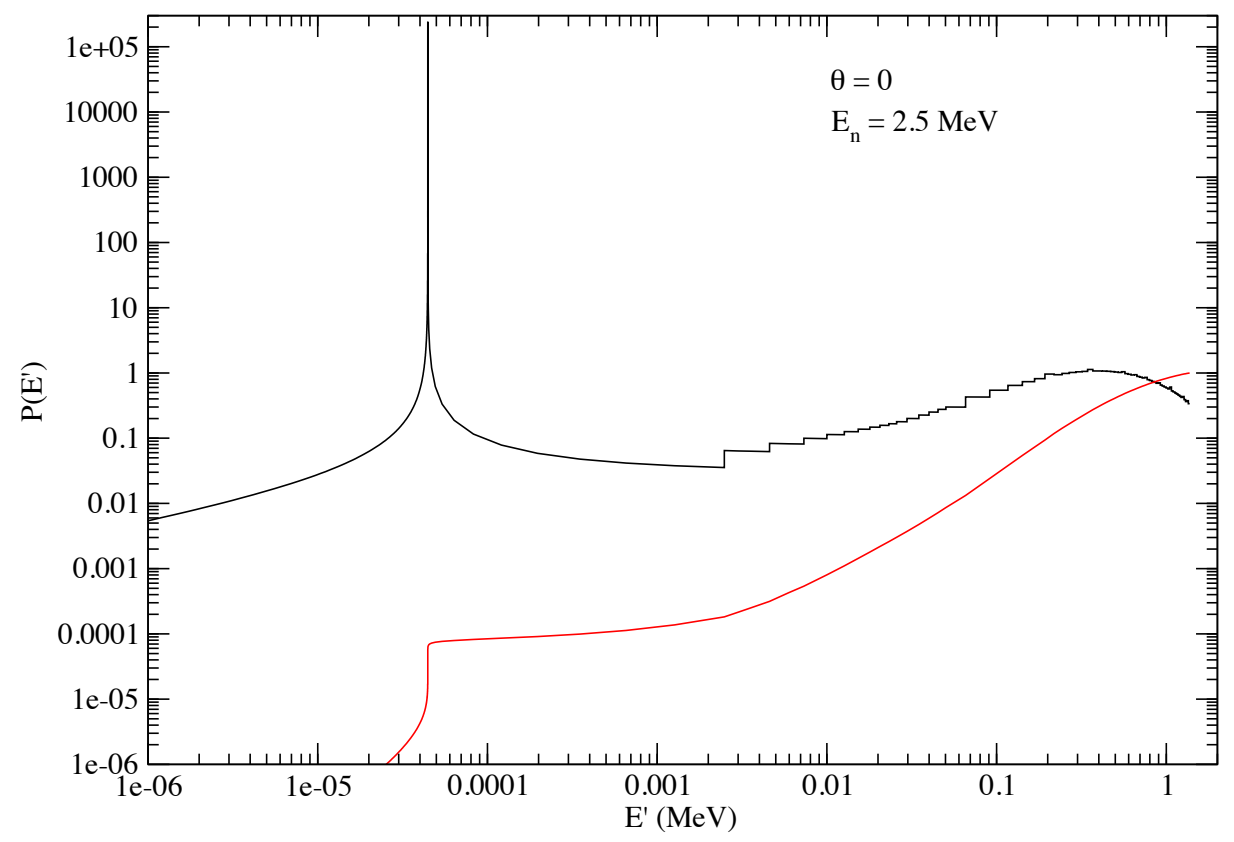

FIG. 16: Emission spectrum probability for forward angles in transitions to unspecified states. In the lower panel is an integral of the probability. 
that have a ${ }^{238} \mathrm{U}$ reflector [24]. These were carried out by Marie-Anne Descalle with the transport code MERCURY [23] There were eleven assemblies: five with highly enriched uranium (HEU; shown in the left of Fig. 17 from Flapttop to HEU_MET_FAST_084_3), one with intermediate enriched uranium (IEU; Bigten), three with plutonium (PU; FlattopPu to PU_MET_FAST_020), and two with ${ }^{233}$ U (U233_MET_FAST_003 to Flattop-23). We performed five simulations with different combinations of data to compute $k_{\text {eff }}$, and the

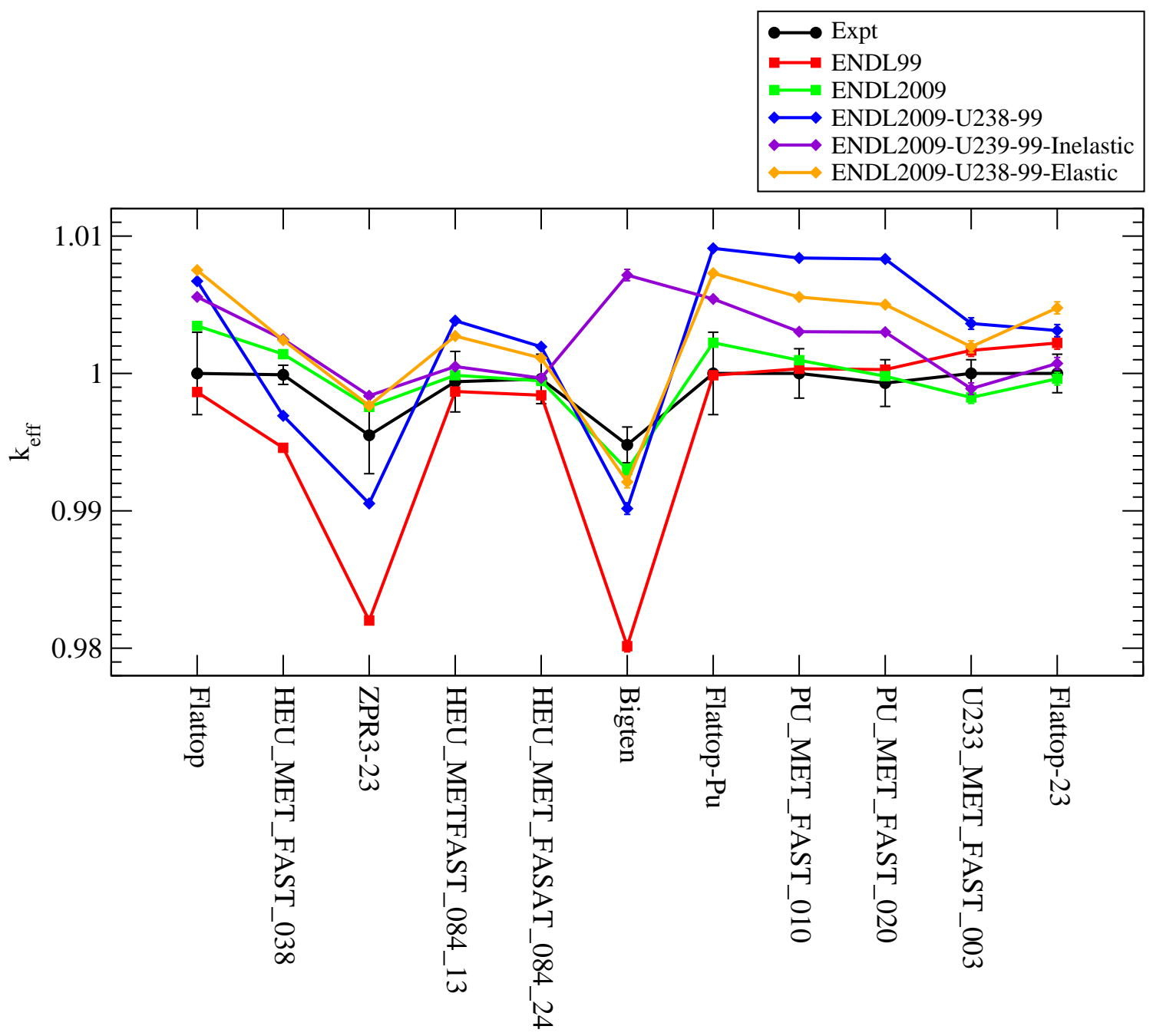

FIG. 17: Plot of measured (black circles) and computed $k_{\text {eff }}$ for twelve critical assemblies constructed with ${ }^{238} \mathrm{U}$ reflectors. Simulations were performed with the ENDL99 (red squares) and ENDL2009 (green squares) libraries as well with replacements in the ENDL2009 library with components from the ENDL99 library; full ${ }^{238} \mathrm{U}$ (blue diamonds) and elastic (orange) and inelastic (purple) ${ }^{238} \mathrm{U}$ data. 
results are shown in Fig. 17. The black circles (connected with black lines), with error bars, are the measured values of $k_{\text {eff }}$. The full ENDL99 (red squares) and ENDL2009 (green squares) libraries are shown. We also performed simulations where we replaced the complete ${ }^{238} \mathrm{U}$ (blue diamonds) and then only the elastic (orange diamonds) and inelastic (purple diamonds) ${ }^{238} \mathrm{U}$ data in the ENDL2009 library with its ENDL99 counterpart using the program FUDGE. The error bars shown for the simulations represent the variance from the Monte Carlo procedure used in MERCURY. Overall, ENDL2009 does the better job in reproducing the experimental $k_{\text {eff }}$. In particular, ENDL99 is rather poor for two assemblies, although it is in exact agreement for two of the $\mathrm{Pu}$ assemblies. We note that in general, when we replace the complete ${ }^{238} \mathrm{U}$ ENDL2009 data with ENDL99, $k_{\text {eff }}$ increases. This is particularly true for the $\mathrm{Pu}$ and ${ }^{233} \mathrm{U}$ assemblies. For HEU assemblies, the situation is mixed, with the increasing in some assemblies, and decreasing in others. The trend shown in the figure tends to remain the same. When we replace either the elastic or inelastic data, $k_{\text {eff }}$ tends to lie between the full ENDL2009 library and the ENDL99 ${ }^{238} \mathrm{U}$ replaced library, which is generally what one would think. This is true for the $\mathrm{Pu}$ and U233 assemblies, but it is definitely not the case for Bigten and some of the HEU assemblies.

\section{CONCLUSIONS}

The primary charge of our effort was to analyze the ENDL99 and ENDL2009 library to assess which library, if any, was a better reflection of reality in describing the elastic and inelastic scattering of neutrons on ${ }^{238} \mathrm{U}$ targets. The primary conclusion is that despite some flaws, the ENDL2009 library, which is based on the ENDF/B-VII.0 evaluation, is generally superior to ENDL99.

In Section VI, we analyzed the elastic channel. The two libraries are quite similar, as seen in Fig. 5, for incident neutron energies less than 1.25 MeV, and if anything, the ENDL99 library describes known data slightly better. Above $1.25 \mathrm{MeV}$, it is apparent that the ENDL99 library is not forward peaked enough as compared with experimental data (as seen in Fig. 6). In this energy range, the ENDL2009 library agrees well with the modern optical potentials developed by Soukhovitskii and in this work. In contrast, the ENDL99 library does not describe the observed diffraction patterns seen in the data very well. Some other differences in the total elastic cross section were observed (see Fig. 3), and again, we judge 
the ENDL2009 library to be better. In the end, the elastic data in the two libraries are fairly similar, with probably the largest difference being in the angular distributions above 1.25-3.0 MeV, for which we would judge ENDL2009 to be better.

In Section VII, we analyze the various components making up the inelastic, or $\left(\mathrm{n}, \mathrm{n}^{\prime}\right)$ channel. The main components are: 1) transitions to known discrete states, 2) transitions to added specified states above $E_{c u t}$, and 3) transitions to unspecified states represented by a continuous level density. For the transitions to known discrete states in ${ }^{238} \mathrm{U}$, both evaluations were based on a coupled-channels calculation including only three discrete states. We point out that this is insufficient to achieve convergence in the coupled-channels procedure. The magnitude of the cross section to these states, however, is in good agreement with existing data due to tuning of the parameters. The ENDL99 library shows a curious feature in that the cross section to the $2^{+}$state decreases and goes to $1 \mathrm{mb}$ at $\approx 5 \mathrm{MeV}$. This is unphysical, and in general, the direct components to discrete states tend to decrease in energy too quickly in the ENDL99 library. Regarding their angular distributions, the scattering probabilities for the $2^{+}$state in ENDL99 are in reasonable agreement with ENDL2009 and our calculations, but above $E_{n}=5 \mathrm{MeV}$ this is inconsequential since the overall cross section mysteriously drops to the unphysical $1 \mathrm{mb}$ level. For the $4^{+}$state, the ENDL99 angular distribution is isotropic, which is not in agreement with the data and ENDL2009. In regards to importance, however, the magnitude of the cross section to this state is roughly half that of the $2^{+}$state. It appears that for other states in the rotational band, a DWBA calculation was performed in ENDL2009 to estimate the direct component. This is not the best approach, as a coupled channels calculation would be better, as performed by us with the FLAP-A2.99d potential. It is worth noting, however, that overall, the cross section to these states is estimated with the coupled-channels calculations to be considerably smaller.

The second primary difference in the inelastic channels between the two libraries is in the transitions to unspecified states. The ENDL99 library is purely isotropic in this regime, while the ENDL2009 library is more forward peaked especially at higher incident neutron energies, greater than 6-7 MeV, where pre-equilibrium emission begins. At lower energies, the ENDL2009 library is more isotropic, but is still clearly more forward peaked.

The final difference between the libraries for inelastic scattering lies in the treatment of the specified states in ENDL2009 above the discrete level cutoff. These states were added and a DWBA calculation was performed to estimate a direct excitation to these states. 
These do not correspond to observed states in ${ }^{238} \mathrm{U}$ and were added in order to account for a slight excess observed in experimental data. The influence of these states is to add approximately $150 \mathrm{mb}$ (at the maximum near $E_{n} \approx 5 \mathrm{MeV}$ ) that is more forward peaked than the compound and pre-equilibrium contributions. It is difficult to assess this other than to say it is a plausible "engineering" approach to account for this missing cross section in the calculations. The need for these states is an indication of limits in current models for the compound and pre-equilibrium components that must be addressed if one is to have greater confidence in our ability to model, and hence evaluate, nuclear data for neutron-induced reactions. It is likely that this could be achieved with newer theoretical developments, such as doorway-state models using structure obtained from RPA or QRPA models. This would require some investment, but should be addressed in future evaluations. The influence of these explicit states in transport models needs to be examined, which could be done using the program FUDGE.

We also note a curious feature in the emission spectrum from $\left(n, n^{\prime}\right)$ reactions to unspecified states. There is a very narrow resonance-like feature at very low energies that is unphysical, and is due to the method used to parameterize the emission spectrum, which then becomes apparent when it is used to express the emission spectrum. On the whole, the total probability to this spurious resonance seems to be fairly small, and perhaps not a serious issue, but it should be fixed in future evaluations.

Lastly, a further check on data quality was performed by simulating and computing $k_{\text {eff }}$ for eleven critical assemblies where ${ }^{238} \mathrm{U}$ was used as a reflector. In this case overall better agreement with experiment was found with ENDL2009. It should be pointed out that these are integral checks, that if passed, do not guarantee that the library is correct. It is nevertheless one of several tests that must be passed to provide confidence. In this regard, ENDL2009 passes somewhat better than does ENDL99.

\section{ACKNOWLEDGEMENTS}

We are grateful to help provided by Marie-Anne Descalle for the simulations of the critical assemblies. This work was performed under the auspices of the U.S. Department of Energy by Lawrence Livermore National Laboratory under contract DE-AC52-07NA27344. Lawrence Livermore National Security, LLC 


\section{Appendix A: Comments on Experimental Data Base}

In this Appendix we make some general remarks on the accuracy and completeness of the experimental data that underlie an evaluation, in particular for the case of interest here, neutron reactions on ${ }^{238} \mathrm{U}$. Some types of data (for example, neutron total cross sections) are reasonably complete, and can be used fairly directly in a cross section evaluation without resort to theoretical calculations. However, more typically, the experimental data are too sparse to describe the full set of quantities needed in an evaluation, and theoretical tools must be used to interpolate and extrapolate the experimental data in energy, as well as to generate cross sections in channels where experimental data are poor or missing. One of the most important of these tools is the optical model, which is used to calculate elastic scattering as well as cross sections and transmission coefficients for absorption of the incident neutron into the compound nucleus. Subsequent evaporation of particles from the compound nucleus is handled by a Hauser-Feshbach calculation, which yields partial cross sections for elastic and inelastic scattering, as well as for other channels if they are open, such as fission. In a statically deformed nucleus such as ${ }^{238} \mathrm{U}$, the optical model must be generalized to the coupled-channels optical model, which in addition to elastic scattering allows calculation of the inelastic scattering to all members of the rotational band of which the target state is a member.

The coupled-channels optical model contains many parameters. Although more fundamental nuclear theory provides useful guidance in determining the general behavior of these parameters, such considerations are unable to provide values of the optical parameters with sufficient accuracy. Thus the parameters must be viewed phenomenologically and their values determined by fitting to the available experimental data base. This leads to the important observation that the accuracy of cross sections predicted by optical model calculations can be no better than the accuracy of the underlying experimental data base.

At very low energies, neutron cross sections show a predominant resonant structure. As the energy rises, the density and width of these resonances increases, they begin to overlap, and at sufficiently high energies the cross section becomes smooth. The quantities calculated by the optical model are slowly-varying energy averages over the fluctuating structure due to the underlying resonances. Some of the optical-model observables, such as the low-energy strength functions mentioned below, are in fact determined by appropriate energy averages 
over measured neutron resonance data.

In the following we briefly characterize the experimental data used in determination of the optical-model parameters for neutrons on ${ }^{238} \mathrm{U}$. Many of these data sets are compared with the present and earlier evaluations in the main part of this report. A more detailed description of the data will be included in a separate report on optical model development.

\section{A.1. Total cross sections}

Neutron total cross sections $\sigma_{\text {tot }}$ are useful because they represent the cross section for the sum of all interaction processes when a neutron strikes a nucleus. Also, with sufficient care they can be measured very accurately $(\approx 1 \%)$ using a sample transmission technique. Above $5.4 \mathrm{MeV}$, we have used the data for ${ }^{238} \mathrm{U}$ measured at the Los Alamos LANSCE/WNR facility [9], which are believed to be accurate to $1 \%$ in the range $5.4-560 \mathrm{MeV}$. In the range 0.050-5.4 MeV, we have relied largely on measurements by Poenitz et al. [7]. There are small disagreements with other measurements in this range $[6,8]$, and we assess the accuracy in this region at $2 \%$.

\section{A.2. Low-energy parameters}

Neutron resonance data measured primarily by sample transmission using continuousenergy ("white") neutron sources from linear accelerators have been appropriately energy averaged to provide the s- and p-wave strength functions $S_{0}$ and $S_{1}$, as well as the potential scattering radius $R^{\prime}$. These quantities are calculable from the optical model. $S_{0}$ and $S_{1}$ are equivalent to the s- and p-wave transmission coefficients, while $R^{\prime}$ is related to the amplitude for the smooth background upon which the s-wave resonances are superimposed. We have used values for these parameters from Mughabghab's Atlas of Neutron Resonances [20], which also contains the definitions of the parameters. Although one might expect a slow variation of these parameters with increasing mass across the actinides, there is a scatter of the order of $20 \%$ in the data for $S_{0}$, which in many cases significantly exceeds the quoted experimental uncertainties. Whether this large scatter originates from experimental problems or variations from nucleus to nucleus due to true physical effects is not well understood. Nevertheless, these data are very important in determining the parameters of the optical 
model at low energies, particularly for the imaginary component of the potential. We require the optical model to reproduce the average behavior of these low-energy parameters, but do not insist on a precise reproduction of the values for a specific nucleus.

\section{A.3. Angular distributions}

Angular distributions of neutrons from elastic scattering as well as inelastic scattering (when experimentally resolved) are among the most important data for determining optical potential parameters. They are normally measured by a time-of-flight technique with a (nearly) mono-energetic pulsed neutron beam. Typical statistical errors for elastic scattering are small at forward angles $(\approx 1 \%)$, and increase to the neighborhood of $10 \%$ at backward angles and in the diffraction minima. Overall normalization errors are typically in the

range $5-10 \%$. In the case of scattering from ${ }^{238} \mathrm{U}$, elastic and inelastic scattering to the $2^{+}$and $4^{+}$members of the ground state rotation band have been measured up to $3.5 \mathrm{MeV}$ incident energy at Bruyeres-le-Chatel [11]. A. B. Smith at Argonne [10] measured elastic scattering in small energy steps from 0.3 to $1.495 \mathrm{MeV}$. In experiments with incident energies above $3.5 \mathrm{MeV}$ the energy resolution was insufficient to resolve the elastic from the inelastic scattering, and the reported angular distributions are for the ground state plus several members of the ground-state rotational band. These data are also useful for optical model development, since such data are essentially equivalent to the angular distribution summed over the entire rotational band, which is also a quantity easily extracted from a coupledchannels optical model calculation. We have used data of this type measured from 4.5 to $10 \mathrm{MeV}$ by Smith [25], and in the 14-MeV region by Hansen et al. [26] and with a larger set of scattering angles by Guanran et al. [27]; these two data sets are in good agreement.

\section{A.4. Absorption}

The absorption or compound-formation cross section $\sigma_{c m p d}$ is critically important because it provides the initial step in compound nuclear reactions. It is calculated from the optical model, and in principle the optical model parameters should be chosen to reproduce a set of measured values of this quantity. Unfortunately, the existing data are very sparse and often unreliable. The principal measurement technique involves determining the transmission of 
neutrons through a spherical shell (see, for example, [28] and references therein). Only a few of these measurements have been made with sufficient attention to various corrections and with sufficient documentation. The work carried out at Livermore in the late 1950's is well documented [28-31] and yields results that appear to be accurate to the level of 3-5\% at energies in the range $7-29 \mathrm{MeV}$, including an extensive survey across the periodic table at $14 \mathrm{MeV}$. Another technique is to obtain $\sigma_{c m p d}$ by subtracting the elastic and direct inelastic cross sections from the total cross section. This method is subject to large errors from the subtraction of large quantities that have been measured in different ways with different systematic errors. The result is that for ${ }^{238} \mathrm{U}, \sigma_{c m p d}$ is probably known to no better than 10-20\%, whereas it would be desirable to know this quantity to $\approx 5 \%$ or better. Improved measurements could probably be carried out with modern techniques using the sphere-transmission method. However, in the absence of new measurements, we must rely on predictions of the optical model with parameters adjusted to fit data on other observables.

\section{A.5. Deformation parameters}

In addition to parameters of the optical model per se, calculations for ${ }^{238} \mathrm{U}$ require a description of the nuclear deformation. The most important of these are the quadrupole and hexadecapole deformations, described by the parameters $\beta_{2}$ and $\beta_{4}$, respectively. We have evaluated these parameters by averaging the results of scattering experiments on ${ }^{238} \mathrm{U}$ using results of available experiments with both neutrons and protons as projectiles. The uncertainty in the most important parameter, $\beta_{2}$, is in the neighborhood of $5-9 \%$, depending on the method of averaging. We have found significant sensitivity to the magnitude of $\beta_{2}$ in some of the optical-model observables in the energy range below about $1.5 \mathrm{MeV}$.

\section{Appendix B: Details for optical potential}

We start with the FLAP2.2 potential of [14, Appendix A], but we modify it because, as mentioned above, the coupled-channels calculations used in its construction were not sufficiently converged. Previously only 3 states of the ground state rotational band were included in the coupled channels calculations for even-even $\left(0^{+}\right)$targets, but now we know we need at least 6 or 7 states for calculations converged to better than $0.1 \%$. The FLAP2.2 
potential has energy-dependent Saxon-Woods parameters defined by linear interpolation between 'energy nodes' taken to be 0, 1, 5, 10, 20 and $50 \mathrm{MeV}$. The resulting Saxon-Woods potential at the beam energy was then deformed according to a standard treatment for the rotational model with quadrupole and hexadecupole deformation parameters $\beta_{2}=0.198$ and $\beta_{4}=0.057$.

The first input step for improving the optical potential is to re-examine the deformation parameters of ${ }^{238} \mathrm{U}$ by using inelastic scattering measurements where these exist. Except for [11], however, all suitable experiments used proton rather than neutron projectiles. We now use deformation lengths $\delta_{k}=\beta_{k} R$, for $R$ the radius of the real volume part, to describe the results of all these experiments. The averaged deformations from experiments [11, 15-19] are $\delta_{2}=1.721 \pm 0.159 \mathrm{fm}$ and $\delta_{4}=0.333 \pm 123 \mathrm{fm}$. There is only weak evidence for a non-zero $\delta_{6}$, so this is set to zero in our calculations.
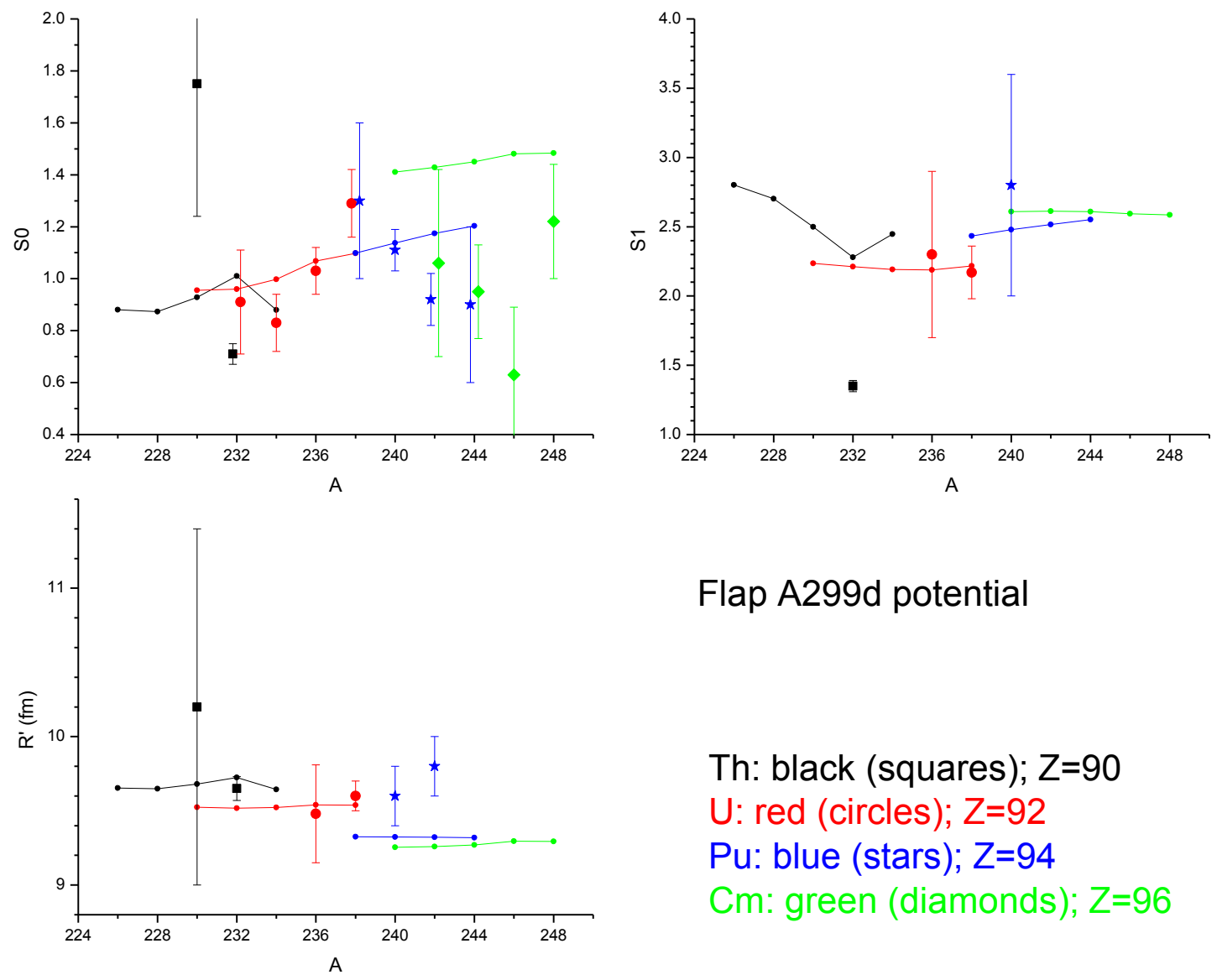

Flap A299d potential

Th: black (squares); Z=90

U: red (circles); $Z=92$

Pu: blue (stars); $Z=94$

Cm: green (diamonds); $Z=96$

FIG. 18: Experimental values [20] and predictions from our FLAP-A2.99d fitted optical potential for the low-energy parameters for neutron scattering on actinide nuclei. 
In neutron scattering at low energies $(E \lesssim 1 \mathrm{MeV})$ there is significant probability of compound-nucleus decay to the elastic channel, so such experimental elastic scattering data cannot be used directly to set the optical potential. The most useful low-energy experiments to fit with an optical potential are therefore the total cross section $\sigma_{T}(E)$ as measured above $50 \mathrm{keV}$, the 'neutron strength functions' $S_{L}$ determined from averages over resonance properties at energies below approximately $1 \mathrm{keV}$ for partial waves $L=0$ and 1 , and the "potential scattering radius' $R^{\prime}$ measured so the $s$-wave background elastic scattering between resonances (i.e., potential scattering) is $\sigma_{\text {pot }}=4 \pi R^{\prime 2}$. For ${ }^{238} \mathrm{U}$, these parameters are measured as $S_{0}=1.29 \pm 0.13, S_{1}=2.17 \pm 0.19$, and $R^{\prime}=9.6 \pm 0.1 \mathrm{fm}$ [20]. The strength function parameters $S_{L}$ are particularly relevant since they are directly related to the CN production cross section at low energies, through the transmission coefficients [20]

$$
\begin{aligned}
T_{L} & =1-\left(1-\pi V_{L} S_{L}\right)^{2} \\
\text { where } \quad V_{0} & =1 \text { and } V_{1}=K^{2} R^{2} /\left(1+K^{2} R^{2}\right),
\end{aligned}
$$

where by convention [20] we use $R=1.35 A^{1 / 3} \mathrm{fm}$ in the construction of the penetrability factors $V_{L}$, and where $K$ is the c.m. wave number of the neutron.

Among the actinides there are some fluctuations in these neutron parameters that cannot be reproduced by smoothly-varying optical potentials, so perfect fits are usually not possible. We, therefore, found our lowest-energy ( $E=0$ node) potential by averaging the parameters to fit the neutron observables for the six nuclei ${ }^{232} \mathrm{Th},{ }^{233,235,238} \mathrm{U}$ and ${ }^{239,240} \mathrm{Pu}$. The values of $V, a$ and $r_{w}$ are fitted as $52.47 \mathrm{MeV}, 0.566 \mathrm{fm}$, and $1.18 \mathrm{fm}$ respectively: changed from their corresponding values in FLAP2.2 of $52.0 \mathrm{MeV}, 0.63 \mathrm{fm}$, and $1.27 \mathrm{fm}$. This gives fits for ${ }^{238} \mathrm{U}$ of $S_{0}=1.05, S_{1}=1.66$, and $R^{\prime}=9.55 \mathrm{fm}$. The predictions for all actinides are shown in Fig. 18.

The parameters for the higher-energy nodes $(E=1,3,5,10$, etc $)$ are then determined at progressively higher energies, since scattering at a given energy is only dependent on the adjacent energy nodes. The most useful data here are the total cross section measurements of Poenitz-1981 [7], Poenitz-1983 [8], and Abfalterer-2001 [9]. At these higher-energy nodes, we retained the geometrical parameters of the original FLAP2.2, and varied the strengths of the real and imaginary central potential components. We also added an additional node at $3 \mathrm{MeV}$. The parameters of the resulting potential, which we call FLAP-A2.99d, are given in a standard notation in Table II. 


\begin{tabular}{lccccccc}
\hline \hline Energy $(\mathrm{MeV})$ & 0 & 1 & 3 & 5 & 10 & 20 & 50 \\
\hline Real Volume & & & & & & & \\
$V_{R 0}$ & 52.470 & 50.614 & 50.614 & 49.845 & 49.856 & 46.810 & 36.433 \\
$V_{R 1}$ & 26.235 & 25.307 & 25.307 & 24.922 & 24.928 & 23.405 & 18.217 \\
$r_{V}$ & 1.250 & 1.260 & 1.260 & 1.260 & 1.240 & 1.230 & 1.210 \\
$a_{V}$ & 0.63 & 0.63 & 0.63 & 0.63 & 0.63 & 0.63 & 0.63 \\
\hline Imaginary $_{\text {Volume }}$ & & & & & & \\
$W_{V 0}$ & 0.000 & 0.000 & 0.000 & 0.000 & 0.305 & 2.143 & 7.557 \\
$W_{V 1}$ & 0.000 & 0.000 & 0.000 & 0.000 & 0.152 & 1.072 & 3.779 \\
$r_{W}$ & 1.270 & 1.270 & 1.270 & 1.270 & 1.270 & 1.270 & 1.270 \\
$a_{W}$ & 0.62 & 0.62 & 0.62 & 0.62 & 0.62 & 0.62 & 0.62 \\
\hline \hline Imaginary Surface & & & & & & \\
$W_{S 0}$ & 3.080 & 2.088 & 3.630 & 4.512 & 6.091 & 6.768 & 1.354 \\
$W_{S 1}$ & 1.540 & 1.044 & 1.815 & 2.256 & 3.045 & 3.384 & 0.677 \\
$r_{S}$ & 1.182 & 1.270 & 1.270 & 1.270 & 1.270 & 1.270 & 1.270 \\
$a_{S}$ & 0.62 & 0.62 & 0.62 & 0.62 & 0.62 & 0.62 & 0.62 \\
\hline \hline
\end{tabular}

TABLE II: Parameters for the FLAP-A2.99d regional actinide optical potential. This is a piecewiselinear potential, so that parameters are to be interpolated linearly between the indicated energies. The strength parameters are given in an isospin representation (subscript 0 for isoscalar, 1 for isovector), which are to be combined as $U=U_{0}-U_{1} \eta$, where $\eta$ is the asymmetry parameter $(N-Z) / A$. Energies are in $\mathrm{MeV}$, and lengths in fm. The spin-orbit potential is the same at all nodes, and is given by $V_{s o 0}=6.993 \mathrm{MeV}, V_{s o 1}=3.497 \mathrm{MeV}, r_{s o}=1.150 \mathrm{fm}$, and $a_{s o}=0.75 \mathrm{fm}$. For ${ }^{238} \mathrm{U}$ it is the same as for FLAP2.2.

Further work is underway to improve the potential, particularly in the $2-5 \mathrm{MeV}$ region. This will be included in a more comprehensive report on the development of the optical potential.

[1] M. Chadwick, et al., Nucl. Data Sheets 107, 2931 (2006). 
[2] M. Chadwick, et al., Nucl. Data Sheets 112, 2887 (2011).

[3] T. Y. Byoun, Experimental investigation of the resonance self-shielding and doppler effect in uranium and tantalum (Rensselaer Polytechnic Institute, Troy, New York, 1973).

[4] J. A. Harvey, N. W. Hill, F. G. Perey, G. L. Tweed, L. Leal, and H. Derrien, Conf.on Nucl.Data For Sci.and Technol.,Mito p. 115 (1988).

[5] Y. V. Grigoriev, V. N. Koshcheev, G. N. Manturov, I. A. Sirakov, V. V. Sinitsa, and N. B. Yaneva, Fiz.-Energ Institut, Obninsk Reports No.2072 (1990).

[6] R. B. Schwartz, R. A. Schrack, and H. T. Heaton, II, Nucl. Sci. Eng. 54, 322 (1974).

[7] W. P. Poenitz, J. F. Whalen, and A. B. Smith, Nucl. Sci. Eng. 78, 333 (1981).

[8] W. P. Poenitz and J. F. Whalen (1983).

[9] W. P. Abfalterer, F. B. Bateman, F. S. Dietrich, R. W. Finlay, R. C. Haight, and G. L. Morgan, Phys. Rev. C 63, 044608 (2001).

[10] A. B. Smith, Nucl. Phys. 47, 633 (1963).

[11] G. Haouat, J. C. L. Lachkar, J. Jary, J. Sigaud, and Y. Patin, Nucl. Sci. Eng. 81, 491 (1982).

[12] F. S. Dietrich, I. J. Thompson, and T. Kawano, Phys. Rev. C 85, 044611 (2012).

[13] E. S. Soukhovitskii, S. Chiba, J.-Y. Lee, O. Iwamoto, and T. Fukahori, J. Phys. G 30, 905 (2004).

[14] J. E. Escher and F. S. Dietrich, Phys. Rev. C 81, 024612 (2010).

[15] L. F. Hansen, I. D. Proctor, D. W. Heikkinen, and V. A. Madsen, Phys. Rev. C 25, 189 (1982).

[16] I. Brissaud, G. Berrier-Ronsin, J. Cameron, R. Frascaria, J. Kalifa, G. Bagieu, and R. de Swiniarski, Zeitschrift für Physik A Hadrons and Nuclei 293, 1 (1979).

[17] J. M. Moss, Y. D. Terrien, R. M. Lombard, C. Brassard, J. M. Loiseaux, and F. Resmini, Phys. Rev. Lett. 26, 1488 (1971).

[18] C. H. King, J. E. Finck, G. M. Crawley, J. A. Nolen, and R. M. Ronningen, Phys. Rev. C 20, $2084(1979)$.

[19] Y. Takeuchi, H. Sakaguchi, M. Nakamura, T. Ichihara, M. Yosoi, M. Ieiri, and S. Kobayashi, Phys. Rev. C 34, 493 (1986).

[20] S. Mughabghab, Atlas of neutron resonances: resonance parameters and thermal cross sections $Z=1-100$ (Elsevier, Amsterdam, 2006).

[21] C. Kalbach, Phys. Rev. C 37, 2350 (1988). 
[22] M. Baba, H. Wakabayashi, N. Ito, K. Maeda, and N. Hirakawa, J. Nucl. Sci. and Tech. 27, 601 (1990).

[23] P. S. Brantley, S. A. Dawson, M. S. McKinley, M. J. O’Brien, R. J. Procassini, S. M. Sepke, and D. E. Stevens, Tech. Rep. LLNL-SM-560687, Lawrence Livermore National Laboratory (2012).

[24] B. Briggs, Tech. Rep. 43 NEA/NSC/DOC(95)03, Nuclear Energy Agency (2010).

[25] A. B. Smith and S. Chiba, Ann. Nucl. Energy 23, 459 (1996).

[26] L. F. Hansen, B. A. Pohl, C. Wong, R. C. Haight, and Ch. Lagrange, Phys. Rev. C 34, 2075 (1986).

[27] S. Guanran, H. Tangzi, W. Shenlin, Y. Chunying, L. Anli, T. Hongqing, S. Qingbiao, Z. Zhixiang, and G. Fuhua, Chinese J. Nucl. Phys. (Beijing) 6, 193 (1984).

[28] M. H. MacGregor, W. P. Ball, and R. Booth, Phys. Rev. 108, 726 (1957).

[29] M. H. MacGregor, W. P. Ball, and R. Booth, Phys. Rev. 111, 1155 (1958).

[30] W. P. Ball, M. H. MacGregor, and R. Booth, Phys. Rev. 110, 1392 (1958).

[31] M. H. MacGregor, R. Booth, and W. P. Ball, Phys. Rev. 130, 147 (1963). 